\title{
Handling missing weak classifiers in boosted cascade: application to multiview and occluded face detection
}

\author{
Pierre Bouges $^{1 *}$, Thierry Chateau ${ }^{1 *}$, Christophe Blanc ${ }^{1}$ and Gaëlle Loosli ${ }^{2}$
}

\begin{abstract}
We propose a generic framework to handle missing weak classifiers at testing stage in a boosted cascade. The main contribution is a probabilistic formulation of the cascade structure that considers the uncertainty introduced by missing weak classifiers. This new formulation involves two problems: (1) the approximation of posterior probabilities on each level and (2) the computation of thresholds on these probabilities to make a decision. Both problems are studied, and several solutions are proposed and evaluated. The method is then applied to two popular computer vision applications: detecting occluded faces and detecting faces in a pose different than the one learned. Experimental results are provided using conventional databases to evaluate the proposed strategies related to basic ones.
\end{abstract}

Keywords: Pattern recognition; Supervised learning; Object detection; Missing data; Adaptation; Face

\section{Introduction}

Boosted cascade is a popular technique in the field of object detection. Boosting algorithms are learning algorithms that combine weak classifiers to produce a strong classifier. A weak classifier is a classifier that is slightly better than random to detect objects. A strong classifier is a classifier which is supposed to have high detection performance. When a candidate area is to be processed, each weak classifier is applied to a part of this area (see Figure 1a). In many computer vision detection applications, the algorithm has to handle partial observations, i.e., the object is partially occluded (see Figure $1 \mathrm{~b}$ ) or has to be detected in a pose different than the one learned (see Figure 1c). In such situations, weak classifiers that are in charge of classifying occluded areas tend to corrupt the final decision, i.e., the candidate area will often be classified as a non-object. Existing solutions consist in defining a set of finite occlusion configurations (or a set of pose configurations) and train multiple boosted cascades, one per configuration (see [1] for an example of multiview face detection). In the proposed solution, multiple training is

\footnotetext{
*Correspondence: pierre.bouges@univ-bpclermont.fr;

thierry.chateau@univ-bpclermont.fr

1 Institut Pascal, Université Blaise Pascal 24, avenue des Landais, Aubière cedex 63177, France

Full list of author information is available at the end of the article
}

avoided (only one classifier is used) and occluded weak classifiers are considered as missing data. A weak classifier is occluded when the data window of the weak classifier has hit an occluded part of the face.

Missing data in classification can be divided into two subproblems: (1) missing data at training stage and (2) missing data at testing stage. In this paper, we assume that missing data only occur at testing stage and that training is done with complete data. A recent study on missing data at testing stage can be found in [2] where Saar-Tsechansky and Provost evaluate different methods to handle missing data at testing stage. They compare two kinds of approach: reduced models and predictive value imputation. Their study does not focus on boosted cascades; the solution we propose in this paper is, to our knowledge, the first algorithm that handles missing data in a boosted cascade without modifying the initial training. Most existing solutions are based on learning algorithms that are designed to be robust to missing data. For example, Smeraldi et al. [3] used a modified version of adaptive boosting (AdaBoost) where weak classifiers can abstain when a feature is missing. Another algorithm was proposed by Globerson and Roweis [4] which is built to be robust to feature deletion. In the same way, Dekel and Shamir [5] improved this idea with an algorithm robust to feature deletion and feature corruption. Chen et al. proposed [6] a solution to

\section{Springer}

(C) 2013 Bouges et al: licensee Springer. This is an Open Access article distributed under the terms of the Creative Commons Attribution License (http://creativecommons.org/licenses/by/2.0), which permits unrestricted use, distribution, and reproduction in any medium, provided the original work is properly cited. 


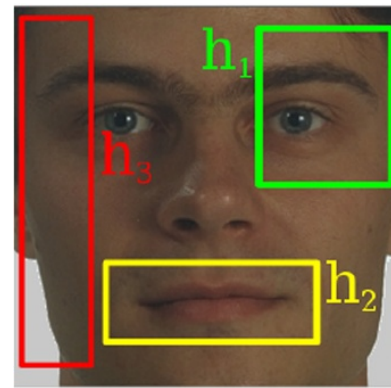

a

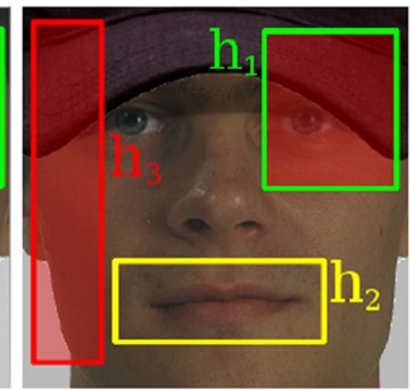

b

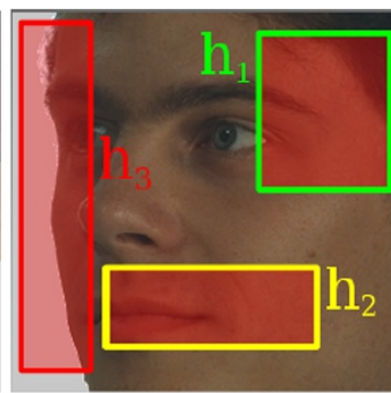

c

Figure 1 Subwindows of weak classifier on an upright face, an occluded face, and a turned face. (a) An example of learned weak classifiers. Each one is in charge of classifying a subwindow. In (b), the face is occluded, and the subwindow of $h_{1}$ and $h_{3}$, filled in red, might be classified as non-face. Similarly, the face in (c) is turned $45^{\circ}$, and all subwindows might be classified as non-face.

detect occluded faces using only one upright face classifier, but they lost the cascade structure resulting in a high detection time.

Here we propose a generic solution to the problem of occluded object detection where occluded weak classifiers are considered as unavailable. Unavailable weak classifiers are seen as missing data, and this fact is incorporated in the cascade structure. We evaluate the proposed method for two different applications: (1) detecting occluded faces and (2) detecting faces in a pose different than the one learned. For each application, we explain how weak classifiers can be considered as available or not. Our method differs from former studies $[1,7]$ in two aspects: the proposed solution does not need the training of multiple classifiers, and, as opposed to existing methods where classifiers are designed to detect objects in a specific pose or with specific occlusions, the proposed solution relies on only one classifier that can adapt to specific poses or occlusions.

Section 2 presents the principle of boosted cascade. A new algorithm that handles missing weak classifiers in a boosted cascade is then detailed in Section 3. Application to occluded faces is presented in Section 4, followed by application to multiview face detection in Section 5. The proposed method is then evaluated in Section 6.

\section{Boosted cascade overview}

This section presents the principle of boosted cascade. The boosting algorithm was introduced by Schapire [8], and many extensions have been proposed. The main idea is to combine the performance of many weak classifiers to produce a powerful strong classifier. The goal is then to perform binary classification. In this paper, we focus on real boosting algorithms (e.g., Real AdaBoost, LogitBoost, or Gentle AdaBoost) which means that weak classifiers are real-valued functions.

Let $\mathcal{L}=\left\{\left(\mathbf{x}_{i}, y_{i}\right)\right\}_{i=1}^{N}$ be a training set where $\mathbf{x}_{i}$ are training examples and $y_{i} \in\{-1,1\}$ are their corresponding labels ( 1 is for the object class, also called positive class). Given this set, a real boosting algorithm iteratively finds $T$ weak classifiers $h_{t}$ to form a strong classifier $\operatorname{sign}(H(\mathbf{x}))=$ $\operatorname{sign}\left(\sum_{t=1}^{T} h_{t}(\mathbf{x})\right)$ where $\mathbf{x}$ is a sample to be classified. Moreover, sign $\left(h_{t}(\mathbf{x})\right)$ gives the label of $\mathbf{x}$ predicted by $h_{t}$, and the value $\left|h_{t}(\mathbf{x})\right|$ represents the confidence of the prediction. Each training example $\mathbf{x}_{i}$ is an image $R_{i}$ of the object or non-object, and each weak classifier $h_{t}$ is learned on a set of subwindows $\left\{r_{t i}\right\}_{i=1}^{N}$ which correspond to discriminative areas in all images $\left\{R_{i}\right\}_{i=1}^{N}$ (see Figure 1a for an example of such subwindows).

To speed up classification, Viola and Jones [9] proposed a cascade structure where several strong classifiers are associated into successive levels. The idea is that the first strong classifiers reject most of the negative examples, while the last strong classifiers try to discriminate positive examples from hard negative examples. In such cascades, strong classifiers are slightly changed into $\operatorname{sign}\left(H_{j}(\mathbf{x})-\right.$ $\left.\alpha_{j}\right)=\operatorname{sign}\left(\sum_{t=1}^{T_{j}} h_{j t}(\mathbf{x})-\alpha_{j}\right)$ where $\alpha_{j}$ are thresholds that are fixed during training (without cascade, $\alpha_{j}=0$ ). The training of a boosted cascade requires five elements: (1) the value $f_{\max }$, the maximum acceptable false-positive rate per level; (2) the value $d_{\text {min }}$, the minimum acceptable detection rate per level; (3) the value $F$, the overall falsepositive rate to be achieved, (4) a set $\mathcal{S}^{p}$ of positive images; and (5) a set $\mathcal{B}$ of background images that will be used to generate interesting negative examples during training. The training of the level $j$ consists of two steps: (1) applying the current cascaded detector (level 1 to $j-1$ ) on $\mathcal{B}$ to generate false-positives and create a set of negative examples $\mathcal{S}^{n}$ and (2) using $\mathcal{S}^{p}$ and $\mathcal{S}^{n}$ to train the strong classifier $\operatorname{sign}\left(H_{j}-\alpha_{j}\right)$. This one is designed so that a detection rate of at least $d_{\min }$ and a false-positive rate of at most $f_{\max }$ are achieved. Both parameters $d_{\min }$ and $f_{\max }$ are fixed by the user. These two steps are repeated until the constraint defined by $F$ is satisfied. In this paper, we consider that the training stage is already done: the cascade of strong classifiers $\left\{\operatorname{sign}\left(H_{1}-\alpha_{1}\right), \ldots, \operatorname{sign}\left(H_{K}-\alpha_{K}\right)\right\}$ is 
available. The following section presents a generic framework to use this cascade when some weak classifiers $h_{j t}$ are missing at testing stage.

\section{Handling missing weak classifiers}

This section presents the problem of missing weak classifiers in a boosted cascade, and solutions to this problem are then detailed. To explain our motivation, suppose we want to detect a face occluded by a scarf. In such a situation, all subwindows located on the lower part of the face will overlap the scarf, and thus all associated weak classifiers will tend to classify these subwindows as nonface. On the other hand, subwindows on the upper part of the face are likely to be classified as face. This is why we propose to consider weak classifiers corresponding to features on the lower part of the face as unavailable. Weak classifiers on the upper part of the face remain available. An example with three weak classifiers is given in Figure 2. In this section, it will be assumed that some weak classifiers are available and some are unavailable. We do not focus on why a weak classifier is available or not. These details will be given in Sections 4 and 5 which are dedicated to occluded face detection and to multiview face detection.

\subsection{Naive approach}

Suppose that we want to classify a sample $\mathbf{x}$ with a strong classifier $\operatorname{sign}(H-\alpha)$ where $H$ is made up of a set of weak classifiers $\left\{h_{1}, \ldots, h_{T}\right\}$. Suppose also that only $p<T$ weak

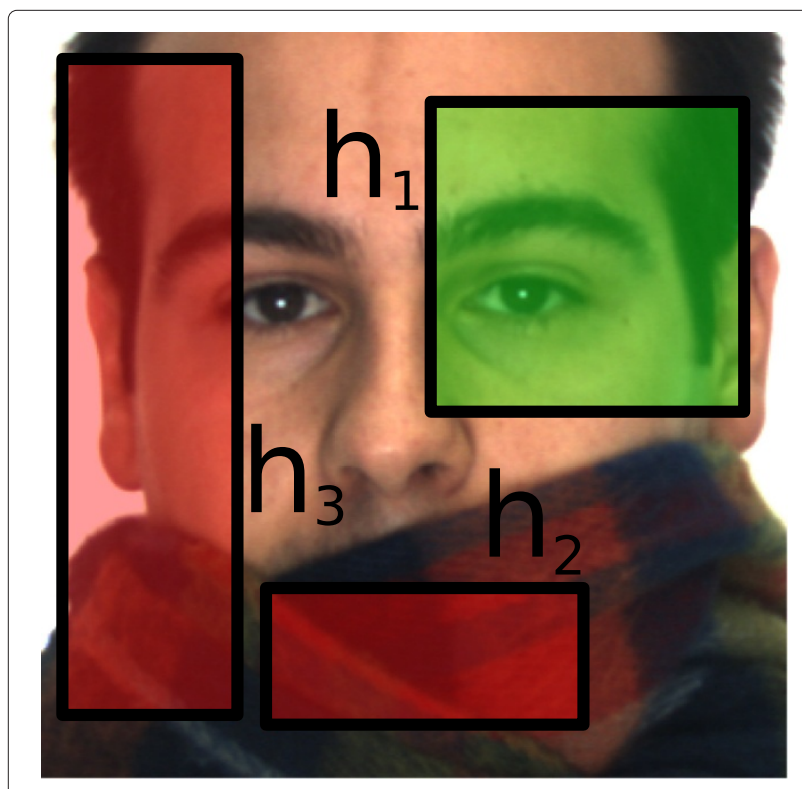

Figure 2 Example of a situation where some weak classifiers are missing. The face is occluded by a scarf. Rather than using all weak classifiers, we propose to use only the weak classifiers that should classify the upper part of the face (in green in the figure). The others, in red, are considered as unavailable. classifiers are available, given by $\left\{h_{\mathrm{a}_{1}}, \ldots, h_{\mathrm{a}_{p}}\right\}$. The set of unavailable weak classifiers is defined as $\left\{h_{\mathrm{u}_{1}}, \ldots, h_{\mathrm{u}_{q}}\right\}$ where $q=T-p$. In such a situation, the easiest strategy to classify $\mathbf{x}$ consists in setting all unavailable weak classifiers to zero, i.e., $h_{u_{1}}(\mathbf{x})=\cdots=h_{u_{q}}(\mathbf{x})=0$. If we note $H_{\mathrm{a}}(\mathbf{x})=$ $\sum_{t=1}^{p} h_{\mathrm{a}_{t}}(\mathbf{x})$, the strong classifier becomes $\operatorname{sign}\left(H_{\mathrm{a}}-\alpha\right)$. By applying this principle to all cascade levels, the set of strong classifiers becomes $\left\{\operatorname{sign}\left(H_{1 \mathrm{a}}-\alpha_{1}\right), \ldots, \operatorname{sign}\left(H_{K \mathrm{a}}-\right.\right.$ $\left.\alpha_{K}\right)$ \}. To sum up, the naive approach consists in setting all unavailable weak classifiers to zero and keeping all cascade thresholds unchanged. This approach will be used as our baseline in the experiments section and will be referred to as 'naive approach'.

\subsection{Probabilistic formulation of a boosted cascade}

In a real boosting algorithm, the predicted label $y \in$ $\{-1,1\}$ of a sample $\mathbf{x}$ can be seen as a discrete random variable and $H(\mathbf{x})$ can be interpreted as the probability of $y$ being an object given the example $\mathbf{x}$ (also called the posterior probability) using the following sigmoid function [10]:

$$
P(y=1 \mid \mathbf{x})=\mathrm{e}^{H(\mathbf{x})} /\left(\mathrm{e}^{H(\mathbf{x})}+\mathrm{e}^{-H(\mathbf{x})}\right) .
$$

Thus, each cascade level computes $P\left(y_{j}=1 \mid \mathbf{x}\right)$ where $y_{j}$ is the predicted label of the level $j$. If a sample $\mathbf{x}$ reaches the level $j$, it means that it has passed all previous levels and is a candidate for an object. This is why we have $P\left(y_{j}=1 \mid \mathbf{x}\right)=P\left(y_{j}=1 \mid \mathbf{x}, y_{1}=1, \ldots, y_{j-1}=1\right)$. When weak classifiers are missing, uncertainty is introduced on each predicted label $y_{j}$. This uncertainty is not considered in the probability $P\left(y_{j}=1 \mid \mathbf{x}, y_{1}=1, \ldots, y_{j-1}=1\right)$ as labels $y_{1}, \ldots, y_{j-1}$ are supposed to be positive. This is why we propose to compute $P\left(y_{1}=1, \ldots, y_{j}=1 \mid \mathbf{x}\right)$ on level $j$. Thus, the predicted label on level $j$ will also depend on predicted labels of level 1 to $j-1$. In the rest of the paper, the event $y_{1}=1, \ldots, y_{j}=1$ will be noted $y_{1: j}=1$ to simplify the notation. To compute $P\left(y_{1: j}=1 \mid \mathbf{x}\right)$, the following rule is used:

$$
P(A, B \mid C)=P(B \mid A, C) P(A \mid C) .
$$

This rule gives:

$$
\begin{aligned}
P\left(y_{1: j}=1 \mid \mathbf{x}\right)= & P\left(y_{j}=1 \mid \mathbf{x}, y_{1: j-1}=1\right) \\
& \times P\left(y_{1: j-1}=1 \mid \mathbf{x}\right) \quad \forall j>1 .
\end{aligned}
$$

By applying this rule recursively, we get:

$$
\begin{aligned}
P\left(y_{1: j}=1 \mid \mathbf{x}\right)= & \prod_{i=2}^{j} P\left(y_{i}=1 \mid \mathbf{x}, y_{1: i-1}=1\right) \\
& \times P\left(y_{1}=1 \mid \mathbf{x}\right) \quad \forall j>1 \\
& =\prod_{i=1}^{j} P\left(y_{i}=1 \mid \mathbf{x}\right) .
\end{aligned}
$$

This probabilistic formulation is very close to the one of Lefakis and Fleuret in [11]. Our motivation remains different because they proposed a new learning algorithm 
based on a probabilistic cascade formulation. In our case, we use a probabilistic formulation to handle the fact that some weak classifiers are missing at testing stage.

In a conventional cascade formulation, each level $j$ applies a strong classifier $H_{j}$ to $\mathbf{x}$ and compares $H_{j}(\mathbf{x})$ with a threshold $\alpha_{j}$. With the probabilistic formulation, all thresholds $\alpha_{j}$ disappear and new thresholds $\beta_{j}$ are introduced. Indeed, we have $P\left(y_{j}=1 \mid \mathbf{x}\right) \leq 1$, and so:

$$
\begin{aligned}
\prod_{i=1}^{j} P\left(y_{i}=1 \mid \mathbf{x}\right) & \leq \prod_{i=1}^{j-1} P\left(y_{i}=1 \mid \mathbf{x}\right) \\
& \leq \cdots \leq P\left(y_{1}=1 \mid \mathbf{x}\right)
\end{aligned}
$$

Equation 6 shows that if $P\left(y_{1: j}=1 \mid \mathbf{x}\right)$ is lower than a value $\beta_{j}$, the cascade process should stop because $P\left(y_{1: j+1}=1 \mid \mathbf{x}\right), \ldots, P\left(y_{1: K}=1 \mid \mathbf{x}\right)$ will be even smaller. In the proposed framework, a strong classifier is defined as $\operatorname{sign}\left(P\left(y_{1: j}=1 \mid \mathbf{x}\right)-\beta_{j}\right)$. The complete modified boosted cascade is then defined by the set of strong clas$\operatorname{sifiers}\left\{\operatorname{sign}\left(P\left(y_{1}=1 \mid \mathbf{x}\right)-\beta_{1}\right), \operatorname{sign}\left(P\left(y_{1: 2}=1 \mid \mathbf{x}\right)-\right.\right.$ $\left.\left.\beta_{2}\right), \ldots, \operatorname{sign}\left(P\left(y_{1: K}=1 \mid \mathbf{x}\right)-\beta_{K}\right)\right\}$. In the following, we refer to this modified cascade as boosted McCascade for boosted cascade with missing classifiers. Figure 3 sums up the differences between a cascade structure and a McCascade structure. Section 3.4 explains how values $\beta_{1}, \ldots, \beta_{K}$ are computed, and the following section focuses on the estimation of $P\left(y_{j}=1 \mid \mathbf{x}\right)$.

\subsection{Posterior probability estimation}

When weak classifiers are missing, the probability $P(y=$ $1 \mid \mathbf{x})$ can no longer be computed and an approximation must be used. We propose three different approximation strategies to do this:

- The simplest strategy to estimate $P(y=1 \mid \mathbf{x})$ is to compute a probability based on available weak classifiers. Thus, we define $P_{\text {boost }}(y=1 \mid \mathbf{x})$ as:

$$
P_{\text {boost }}(y=1 \mid \mathbf{x}) \doteq \mathrm{e}^{H_{\mathrm{a}}(\mathbf{x})} /\left(\mathrm{e}^{H_{\mathrm{a}}(\mathbf{x})}+\mathrm{e}^{-H_{\mathrm{a}}(\mathbf{x})}\right) .
$$

- A second strategy, noted $P_{\mathrm{knn}}(y=1 \mid \mathbf{x})$, tries to benefit from the initial training. Indeed, each training example $\mathbf{x}_{i}$ provides a set of weak classifier values $h_{\mathbf{x}_{i}}=\left(h_{1}\left(\mathbf{x}_{i}\right), \ldots, h_{T}\left(\mathbf{x}_{i}\right)\right)$ and an associated label $y_{i}$. All these weak classifier values form a set $\mathcal{H}=\left\{\left(h_{\mathbf{x}_{i}}, y_{i}\right)\right\}_{i=1}^{N}$, and the subset of available weak classifiers form $\mathcal{H}_{\mathrm{a}}=\left\{\left(h_{\mathrm{ax}_{\mathrm{i}}}, y_{i}\right)\right\}_{i=1}^{N}$ where $h_{\mathrm{a}_{\mathbf{x}_{i}}}=\left(h_{\mathrm{a}_{1}}\left(\mathbf{x}_{i}\right), \ldots, h_{\mathrm{a}_{p}}\left(\mathbf{x}_{i}\right)\right)$. The resulting set $\mathcal{H}_{\mathrm{a}}$ is used as a training set to approximate $P(y=1 \mid \mathbf{x})$ with the help of the $k$-nearest neighbor $(k$-nn) algorithm. Given a sample $\mathbf{x}$, its associated available weak classifier scores $h_{\mathrm{a}_{\mathbf{x}}}=\left(h_{\mathrm{a}_{1}}(\mathbf{x}), \ldots, h_{\mathrm{a}_{p}}(\mathbf{x})\right)$ are first computed. Then, the $k$-nn algorithm searches the $k$ nearest neighbors of the point $h_{\mathrm{a}_{\mathrm{x}}}$ in the space $\mathcal{H}_{\mathrm{a}}$. Considering the labels $\left\{y_{1}^{*}, \ldots, y_{k}^{*}\right\}$ of the $k$ nearest neighbors, the probability $P_{\mathrm{knn}}(y=1 \mid \mathbf{x})$ is computed as:

$$
P_{\mathrm{knn}}(y=1 \mid \mathbf{x}) \doteq \sum_{i=1}^{k} \frac{\mathbb{1}_{\left\{y_{i}^{*}=1\right\}}}{k},
$$

where $1_{\text {pred }}=1$ if the predicate (pred) is true and $\mathbb{1}_{\text {pred }}=0$ otherwise. Figure 4 illustrates the computation of $P_{\text {knn }}(y=1 \mid \mathbf{x})$ when two weak classifiers are available.

- An additional strategy, noted $P_{\text {comb }}(y=1 \mid \mathbf{x})$, consists in combining the two previous methods as the simplest way:

$$
P_{\text {comb }}(y=1 \mid \mathbf{x}) \doteq \frac{P_{\text {boost }}(y=1 \mid \mathbf{x})+P_{\text {knn }}(y=1 \mid \mathbf{x})}{2} .
$$

\subsection{Boosted McCascade threshold estimation}

Before a McCascade can be used to classify a sample $\mathbf{x}$, the threshold $\beta_{1}, \ldots, \beta_{K}$ must be estimated. The threshold $\beta_{1}, \ldots, \beta_{K}$ estimation can be seen as the training stage of a McCascade. This is achieved through an iterative procedure which uses sets $\mathcal{S}^{p}$ and $\mathcal{B}$ from the initial training stage. This procedure is described in Algorithm 1. At iteration $j$, the threshold $\beta_{j}$ of the level $j$ is computed using the following scheme: all probabilities $p_{j i} \doteq P\left(y_{1: j}=1 \mid \mathbf{x}_{i}\right)$ are first computed. Then, the set of probabilities $\left\{p_{j i}\right\}_{i=1}^{N}$ is sorted and $\beta_{j}$ is chosen among the set of finite values $\tilde{p}_{j i} \doteq 0.5\left(p_{j i}+p_{j(i+1)}\right), i \in\{1, \ldots, N-1\}$. The function find_optimal_threshold (see line 14) finds the threshold that minimizes a cost function defined on falsepositive and true-positive rates. Contrary to the initial cascade where each level ensures reaching a true-positive rate of at least $d_{\min }$ with a false-positive rate less than $f_{\max }$, the McCascade cannot guarantee the same performance. The cost function's goal is to ensure that each threshold found provides a performance close to the initial cascade performance. Three cost functions are proposed:

- FP_cost is defined on the false-positive rate $f_{\beta}$ associated to a threshold $\beta$ :

$$
\mathrm{FP} \_\operatorname{cost}\left(f_{\beta}\right) \doteq \max \left(0, f_{\beta}-f_{\max }\right) \text {. }
$$

The false-positive rate $f_{\beta}$ is computed on the training examples. Using this function means that the threshold found provides a false-positive rate which is as close as possible to $f_{\max }$ (it remains greater or equal to $f_{\max }$ ).

- TP_cost is defined on the true-positive rate $d_{\beta}$ associated to a threshold $\beta$ :

$$
\text { TP_cost }\left(d_{\beta}\right) \doteq \max \left(0, d_{\text {min }}-d_{\beta}\right) .
$$

The true-positive rate $d_{\beta}$ is computed on the training examples. The threshold computed with this 


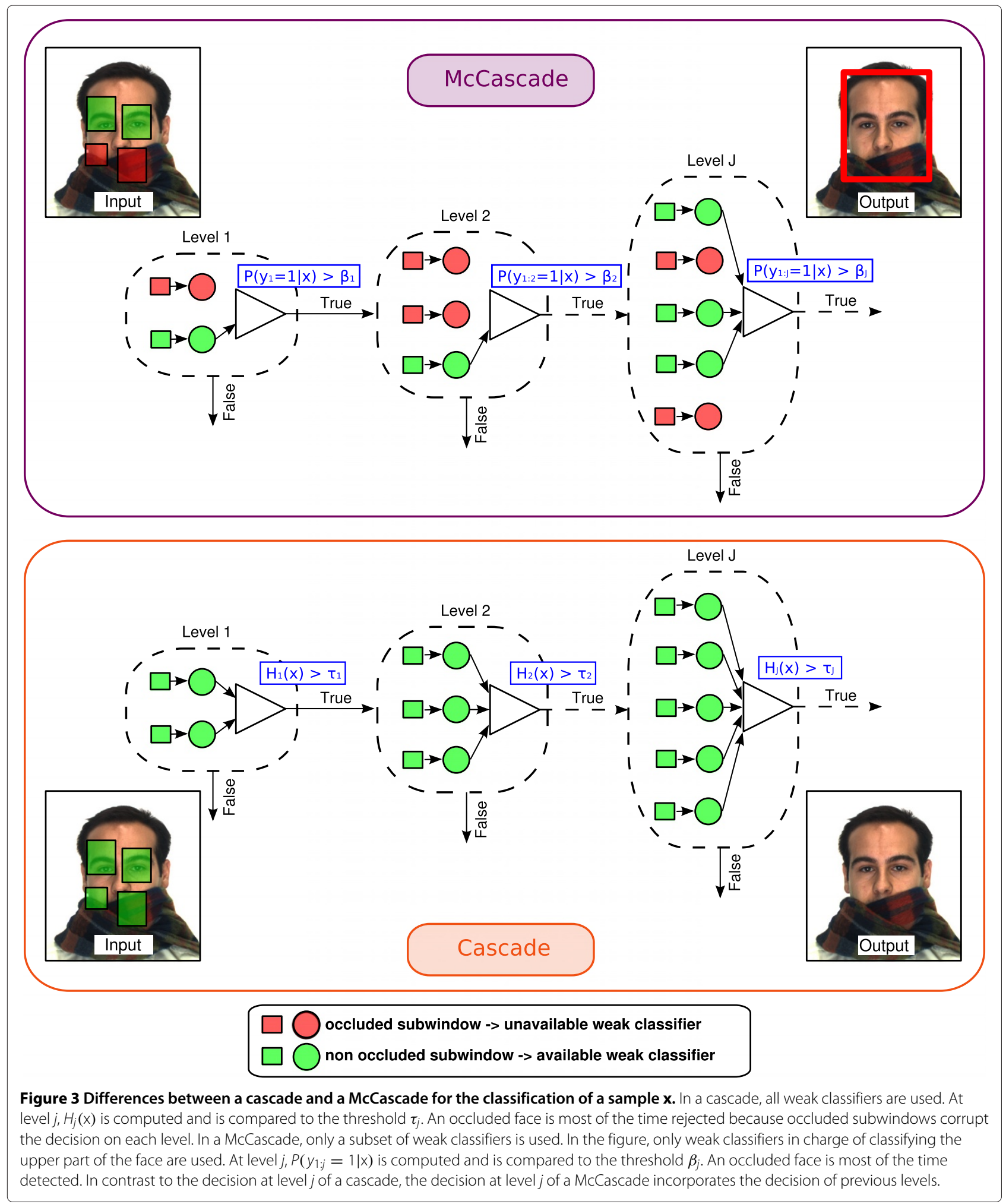

function will ensure a true-positive rate close to $d_{\min }$ (it remains lower or equal to $d_{\min }$ ).

- FP_TP_cost is defined on both false-positive and true-positive rates:
FP_TP_cost $\left(f_{\beta}, d_{\beta}\right) \doteq \mathrm{FP} \_\operatorname{cost}\left(f_{\beta}\right)+\mathrm{TP} \_\operatorname{cost}\left(d_{\beta}\right)$.

This last cost function is a compromise between a false-positive rate of $f_{\max }$ and a true-positive rate of $d_{\text {min }}$. 


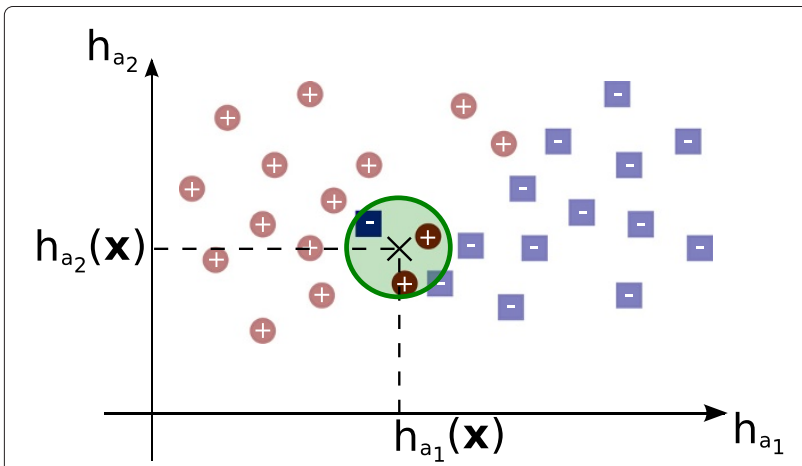

Figure $\mathbf{4}$ Computation of $\boldsymbol{P}_{\mathbf{k n n}}(\boldsymbol{y}=\mathbf{1} \mid \mathbf{x})$. Two weak classifiers are available: $h_{\mathrm{a}_{1}}$ and $h_{\mathrm{a}_{2}}$. Applying these weak classifiers on a training database gives the set of points $\mathcal{H}_{\mathrm{a}}$ (the red circles are positive points and the blue squares are negative points). Given an unknown sample $\mathrm{x}, h_{\mathrm{a}_{1}}(\mathrm{x})$ and $h_{\mathrm{a}_{2}}(\mathrm{x})$ are computed and the $k$ nearest neighbors of $\left(h_{\mathrm{a}_{1}}(\mathrm{x}), h_{\mathrm{a}_{2}}(\mathrm{x})\right)$ are searched in $\mathcal{H}_{\mathrm{a}}$. In the figure, $k=3$, and the nearest neighbors are two positive points and one negative point which leads to $P_{\mathrm{knn}}(y=1 \mid \mathrm{x})=2 / 3$.

A detailed version of find_optimal_threshold with the cost function FP_TP_cost is given in Algorithm 2. Once all the thresholds $\beta_{1}, \ldots, \beta_{K}$ are estimated, the McCascade can be used to classify any unknown sample $\mathbf{x}$.

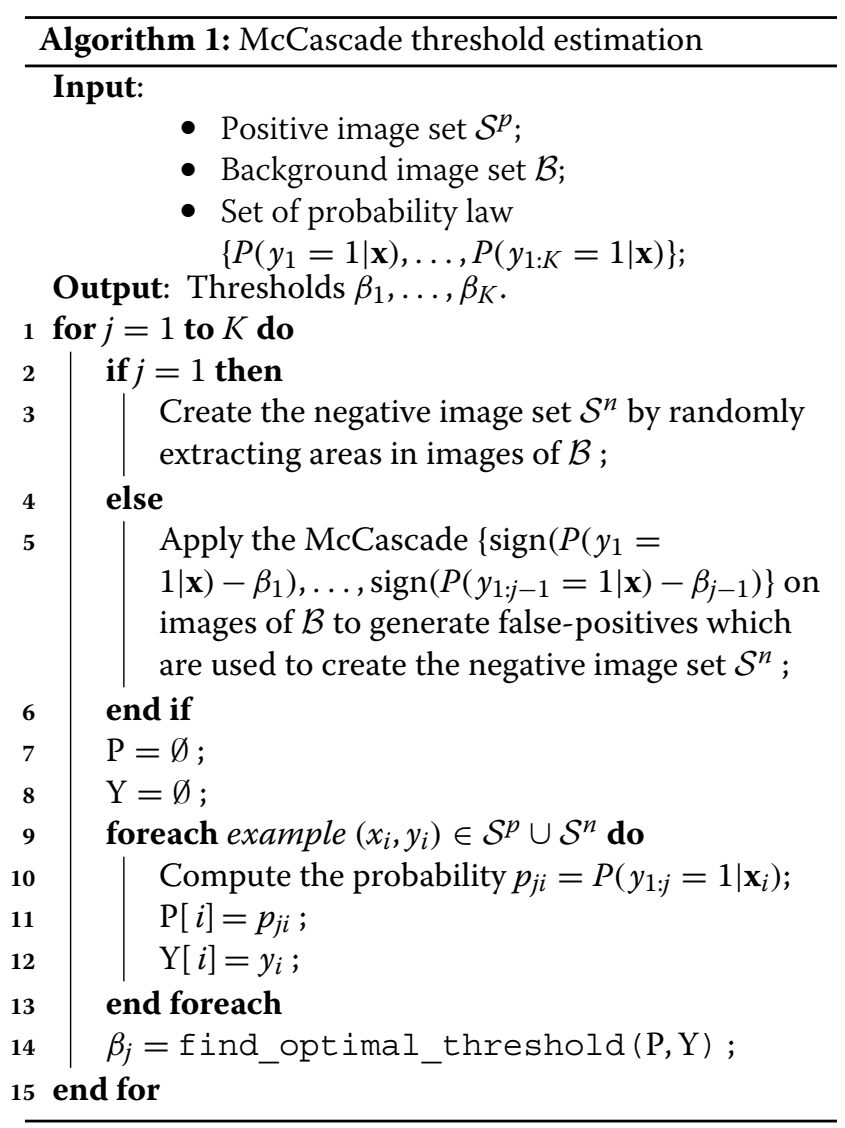

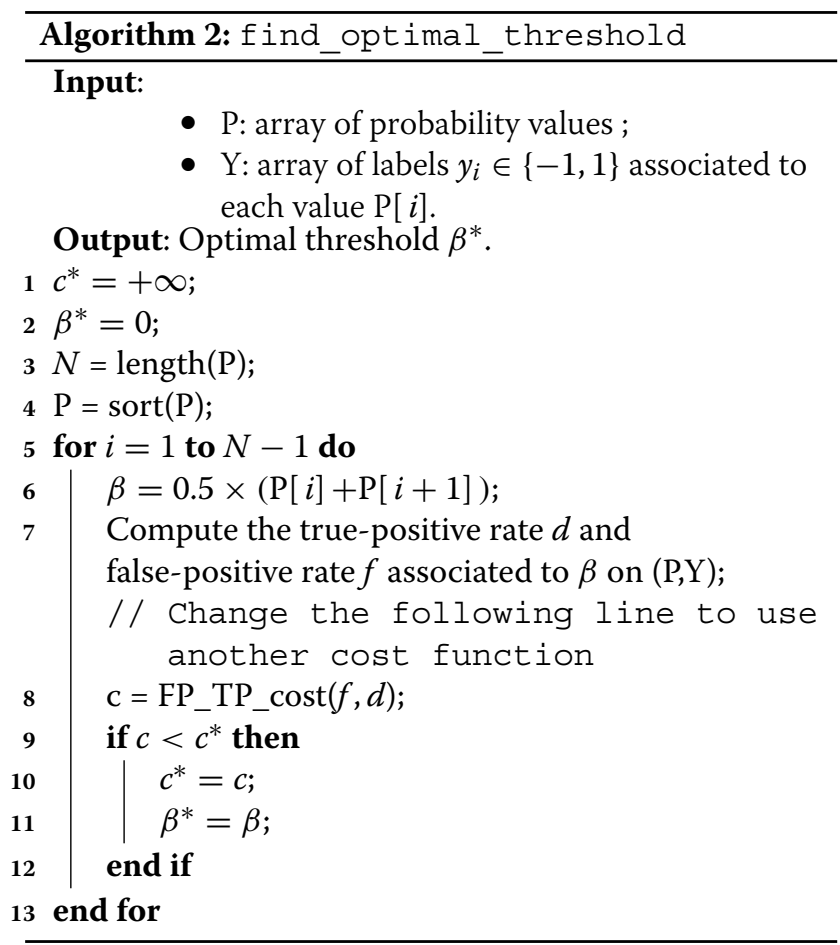

\subsection{Cascade and McCascade training time}

When a McCascade is created, the threshold $\beta_{1}, \ldots, \beta_{K}$ must be computed. This step can be seen as the training stage of a McCascade. Compared to the training stage of a cascade, a McCascade needs fewer time to be trained. The training time of a cascade depends on a lot of parameters: number of training samples, number of levels, implementation (C++/MATLAB), ... Rather than giving precise training times to compare a cascade and a McCascade, rough estimates are given here to emphasize the fact that a McCascade is faster to train than a cascade.

The training stage of a cascade can be split into three steps:

1. Gather training data. Training data are made up of the positive images and of the background images. This step can last a few seconds if a public database exists. It can also last a few days if images must be manually gathered.

2. Generate false-positives. At the beginning of each level, the negative samples are generated by applying the current classifier to the set of the background images. This step can last a few seconds to a few minutes.

3. Train a cascade level. At each boosting iteration, several weak classifiers are learned (one for each subwindow), and the best one is kept. The number of iteration depends on the classification performance that must be reached. This step can last a few minutes to a few hours. 
The training stage of a McCascade can be split into two steps:

1. Generate false-positives. At the beginning of each level, the negative samples are generated by applying the current classifier to the set of the background images. This step can last a few seconds to a few minutes.

2. Fix the level threshold. A probability is computed for each training example, and the threshold is computed according to these probabilities. This step can last a few milliseconds to a few seconds.

An object detector trained with a cascade is designed to detect the object in a specific pose or with specific occlusion. When the object has to be detected in a new pose or with new occlusion, a new object detector has to be designed. Using a cascade means that the three steps must be done again. On the opposite, using a McCascade just requires two steps that are not so time consuming. This is illustrated in Figure 5.

\section{Application to occluded face detection}

Occlusions can greatly change the appearance of a face, and an upright face detector will easily fail to detect such faces. A cascaded detector that can deal with occlusions has already been proposed by Lin et al. [7]. Their solution relies on the training of nine cascaded detectors (one main cascade + eight occlusion cascades) that are then combined. This solution exhibits good performance at the cost of a prohibitive training time. On the other hand, Chan et al. [6] also proposed a detector to handle occlusion with only one training. They first train a boosted

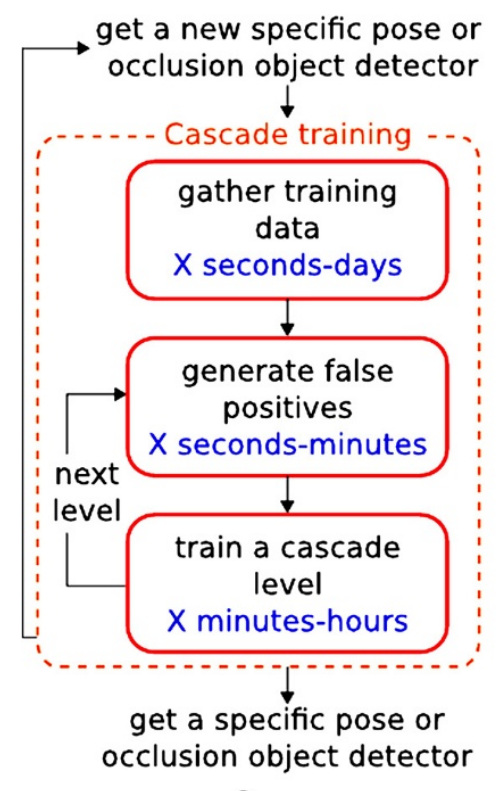

a

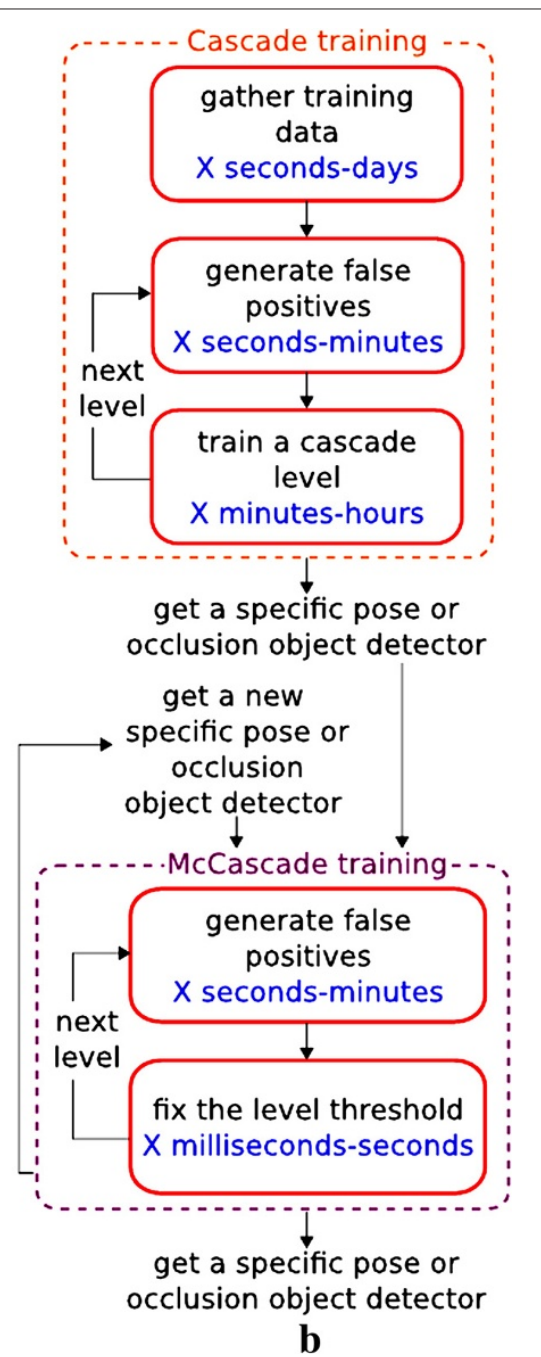

Figure 5 Differences between a cascade and a McCascade when several object detectors must be created. A rough estimate of the execution time is given for each step of the training process. (a) A new cascade must be trained for each new object detector. Each new training can last several hours to several days. (b) A new object detector can be trained using a McCascade. Each training just lasts several minutes. 
cascade and then combine all the weak classifiers learned to obtain a detector robust to occlusions. The problem is that the cascade structure is lost, resulting in an extensive execution time. Our solution relies on the use of an upright face detector $\mathcal{C}$ and the definition of several occlusion configurations where each occlusion configuration is associated with a McCascade. Each occlusion configuration is associated with a set of occluded weak classifiers from all the weak classifiers of the upright face detector. Based on this set, a McCascade that uses nonoccluded weak classifiers can be built. Each McCascade created is called an occlusion cascade. Hence, we build several occlusion cascades which are then combined with the principle of cascading with evidence explained later.

\subsection{Occlusion cascade creation}

Several occlusion cascades are created. Each one is in charge of a given occlusion type. To limit complexity, the case of two occlusion types is presented: bottom occlusion (called type $\mathcal{A}$ in Figure 6a) and top occlusion (called type $\mathcal{B}$ in Figure 6b). In occlusion $\mathcal{A}$, the lower third of the face is considered as occluded. In occlusion $\mathcal{B}$, the upper third of the face is considered as occluded.

Let $\mathcal{O}_{\mathcal{I}}$ be the occluded area with $\mathcal{I} \in\{\mathcal{A}, \mathcal{B}\}$, the set of occlusion configurations. Let $\mathcal{S}_{j t}$ be the region covered by the subwindow associated with the weak classifier $h_{j t}$ (see Figure 7). For each occlusion type $\mathcal{I}$, the set of available weak classifiers must be defined to build the associated occlusion cascade. A weak classifier $h_{j t}$ is available for occlusion $\mathcal{I}$ if the area $\mathcal{S}_{j t}$ does not intersect $\mathcal{O}_{\mathcal{I}}$. In other words, the associated subwindow is considered as occluded for the occlusion $\mathcal{I}$ if the area $\mathcal{S}_{j t}$ intersects $\mathcal{O}_{\mathcal{I}}$. For $\mathcal{I} \in\{\mathcal{A}, \mathcal{B}\}$, two sets $\mathcal{H}^{\mathcal{A}}$ and $\mathcal{H}^{\mathcal{B}}$ of available weak classifiers are defined:

$$
\begin{aligned}
& \mathcal{H}^{\mathcal{A}}=\left\{h_{j t} \mid \mathcal{S}_{j t} \cap \mathcal{O}_{\mathcal{A}}=\emptyset\right\}, \\
& \mathcal{H}^{\mathcal{B}}=\left\{h_{j t} \mid \mathcal{S}_{j t} \cap \mathcal{O}_{\mathcal{B}}=\emptyset\right\} .
\end{aligned}
$$

Based on these two sets, two McCascades $\mathcal{C}^{\mathcal{A}}$ and $\mathcal{C}^{\mathcal{B}}$ can be created. $\mathcal{C}^{\mathcal{A}}$ only uses weak classifiers defined in $\mathcal{H}^{\mathcal{A}}$. In the same way, $\mathcal{C}^{\mathcal{B}}$ only uses weak classifiers defined in $\mathcal{H}^{\mathcal{B}}$. Finally, thresholds $\beta_{j}$ of both McCascades are fixed with the help of Algorithm 1.

\subsection{Cascading with evidence}

To combine the main cascade $\mathcal{C}$ and the two occlusion cascades $\mathcal{C}^{\mathcal{A}}$ and $\mathcal{C}^{\mathcal{B}}$, the principle of cascading with evidence proposed by Lin et al. [7] is used. When a sample $\mathbf{x}$ must be tested, it first goes through the main cascade. At level $j$ of this cascade, in addition to applying the strong classifier $H_{j}$, an additional feature vector $\varepsilon_{j}(\mathbf{x})$ is also computed:

$$
\varepsilon_{j}(\mathbf{x})=\left(H_{j}^{\mathcal{A}}(\mathbf{x}), H_{j}^{\mathcal{B}}(\mathbf{x})\right),
$$

where

$$
H_{j}^{\mathcal{I}}(\mathbf{x})=\sum_{t \mid \mathcal{S}_{j t} \cap \mathcal{O}_{\mathcal{I}}=\emptyset} h_{j t}(\mathbf{x}) \text { with } \mathcal{I} \in\{\mathcal{A}, \mathcal{B}\} .
$$

The vector $\varepsilon_{j}(\mathbf{x})$ is called the evidence of $\mathbf{x}$ at level $j$.

Equation 16 means that $H_{j}^{\mathcal{I}}$ only involves weak classifiers over subwindows that do not intersect with $\mathcal{O}_{\mathcal{I}}$. With the evidence vector presented in Equation 15, weak classifiers can now be defined as available or not depending on the occlusion encountered. Indeed, let $\mathbf{x}$ be an occluded face example of type $\mathcal{A}$ and suppose that the main cascade $\mathcal{C}$ rejects it at level $j$ because $H_{j}(\mathbf{x})<\alpha_{j}$. Before rejecting it, we check the evidence vector of $\mathbf{x}$. In particular, the majority of $H_{1}^{\mathcal{A}}(\mathbf{x}), \ldots, H_{j}^{\mathcal{A}}(\mathbf{x})$ should be positive, indicating that $\mathbf{x}$ is an occluded face of type $\mathcal{A}$. Based on this fact, weak classifiers that can handle occlusion $\mathcal{A}$ (i.e., $h_{j t}$ verifying $\left.\mathcal{S}_{j t} \cap \mathcal{O}_{\mathcal{A}}=\emptyset\right)$ are defined as available, and $\mathbf{x}$ continues the classification process with the McCascade $\mathcal{C}^{\mathcal{A}}$ defined on available weak classifiers. Generally speaking, if a sample is occluded of type $\mathcal{I}$ and if this sample is rejected by the main cascade, this sample will be passed to the McCascade $\mathcal{C}^{\mathcal{I}}$. Note that with this principle of cascading with evidence, there is no explicit occlusion detection.

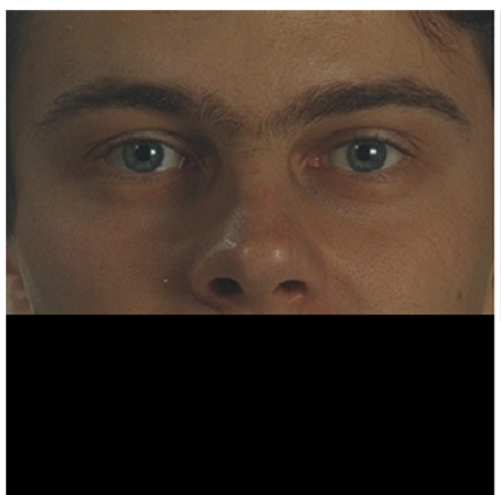

a

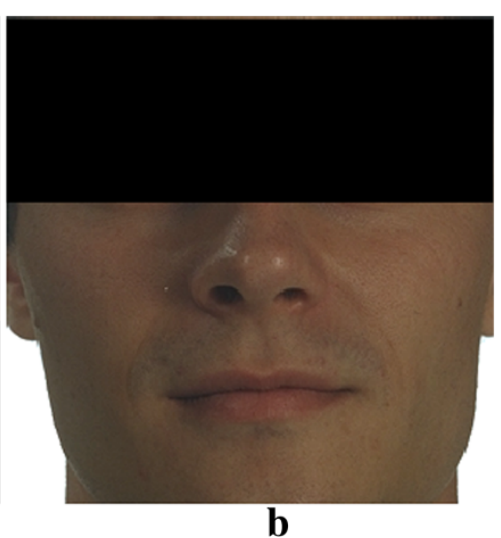

Figure 6 Definition of two occluded areas (one-third occlusion). (a) An example of type $\mathcal{A}$ occluded face. (b) An example of type $\mathcal{B}$ occluded face. 


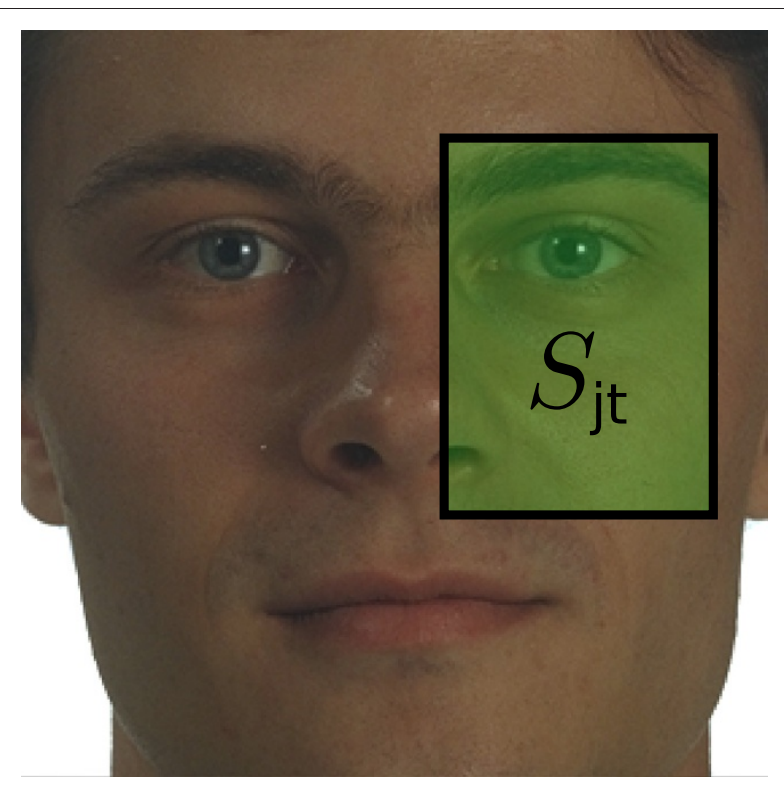

Figure 7 Region covered by the subwindow associated to a weak classifier. The weak classifier $h_{j t}$ must classify the region $\mathcal{S}_{j t}$, filled in green.

Using $\mathcal{C}, \mathcal{C}^{\mathcal{A}}$, and $\mathcal{C}^{\mathcal{B}}$ with the principle of cascading with evidence, we can detect occluded faces following the testing procedure described in Algorithm 3 where $\mathcal{C}^{\mathcal{I}}$ represents the McCascade that can handle occlusion $\mathcal{I}$. The testing procedure is also illustrated in Figure 8. All the above explanations remain valid with other types of occlusions. Note that the number of occlusions that can be handled only depends on the weak classifiers learned during the initial training. For example, if all the weak classifiers learned are associated with subwindows located on the upper part of the face, it would be impossible to handle occlusions of type $\mathcal{B}$.

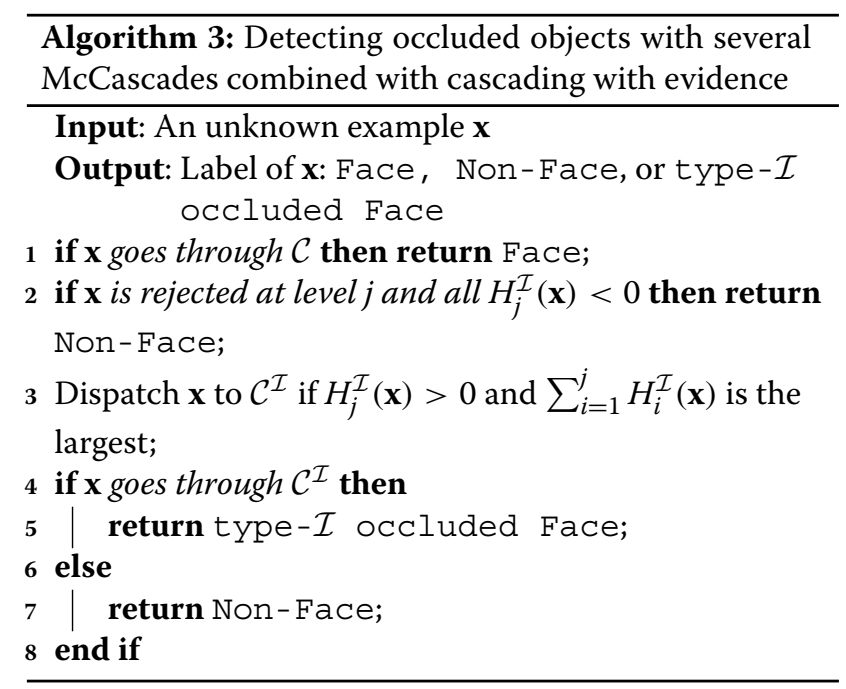

\section{Application to multiview face detection}

In this section, we are interested in the detection of faces with rotation-off-plane (ROP) angles. Examples of such faces are exposed in Figure 9. Upright face detectors are robust to slight ROP angles (they can usually detect faces turned up to $\pm 20^{\circ}$ ). Detection of faces with bigger ROP angles need specific solutions. Most of the existing methods adopt the view-based approach: several classifiers are trained and then combined to get a multiview face detector $[1,12,13]$. In such an approach, each classifier is trained to detect faces with ROP angles in a given range which means that multiple training is necessary. To avoid these multiple trainings, we propose to create a classifier that can detect faces in a pose different than the one learned.

\subsection{Detecting faces with ROP angle}

Our solution is composed of an upright face detector that we modify to be able to detect faces with a given ROP angle. To incorporate the fact that faces may have out-of-plane rotations, we propose to adjust all the subwindow positions. Our idea is illustrated in Figure 10c. Figure 10a shows three interesting subwindows used to detect upright faces. In Figure 10b, we represent the same subwindows on a face turned $45^{\circ}$. The three subwindows are not anymore informative. To alleviate this problem, we can modify the position of the three subwindows (see Figure 10c). Note that the position modification can lead to a modification of the subwindow size (see the yellow subwindow) or the disappearance of some subwindows (see the red subwindow).

To modify a subwindow position, we propose to use the three-dimensional (3D) transformation which exists between an upright face and the same face in another pose. In our case, these transformations are the set of rotations around the $x$-axis and $y$-axis. To simulate a rotation, we need a $3 \mathrm{D}$ face model. Building an accurate 3D face model requires at least two images per face. As our intention is to avoid gathering images other than upright faces, we decide to represent a face with the simplest model: an ellipsoid. The idea is then to place each subwindow on the ellipsoid, turn the ellipsoid, and finally get back all the new subwindows positions. Let us consider a point $p_{1}^{i}=\left(u_{1} v_{1}\right)^{T}$ of an image of size $w \times w$ (the same size as training images) whose coordinates are expressed in the image coordinate system $\mathcal{C} \mathcal{S}_{i}$. The process to compute the position of this point after a rotation defined by an angle of $\theta_{x}$ around the $x$-axis and an angle of $\theta_{y}$ around the $y$-axis is made up of the following three steps:

1. We associate a point $P_{1}^{i}=\left(u_{1} v_{1} w_{1}\right)^{T}$ to the point $p_{1}^{i} . P_{1}^{i}$ is the $3 \mathrm{D}$ point with the same $x$-coordinate and $y$-coordinate as $p_{1}^{i}$ that belongs to the ellipsoid. We just have to compute the $z$-coordinate $w_{1}$ with 


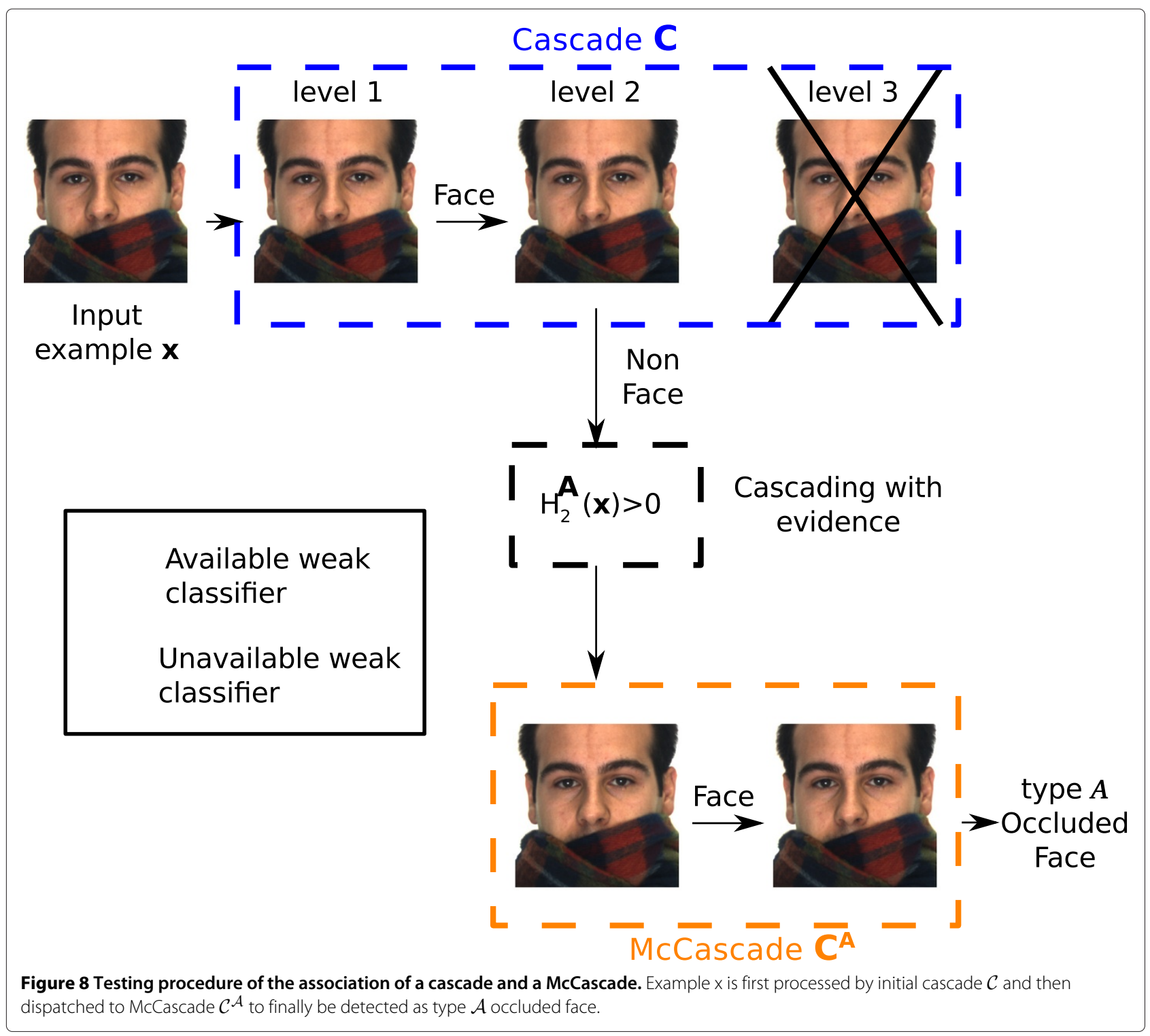

the help of the ellipsoid equation expressed in $\mathcal{C S}_{i}$ (see Figure 11a):

$$
\frac{\left(u-u_{0}\right)^{2}}{a^{2}}+\frac{\left(v-v_{0}\right)^{2}}{b^{2}}+\frac{\left(w-w_{0}\right)^{2}}{c^{2}}=1,
$$

where $u_{o}=w / 2, v_{o}=w / 2$, and $w_{o}=0$ and $a, b$, and $c$ are the ellipsoid's parameters.

2. We express $P_{1}^{i}$ in the coordinate system $\mathcal{C} \mathcal{S}_{e}$ whose origin is the ellipsoid center. This gives us the $P_{1}^{e}$ point:

$$
\left[\begin{array}{c}
\tilde{x}_{1} \\
\tilde{y}_{1} \\
\tilde{z}_{1} \\
\tilde{d}_{1}
\end{array}\right]=\left[\begin{array}{cccc}
1 & 0 & 0 & -w / 2 \\
0 & 1 & 0 & -w / 2 \\
0 & 0 & 1 & 0 \\
0 & 0 & 0 & 1
\end{array}\right]\left[\begin{array}{c}
u_{1} \\
v_{1} \\
w_{1} \\
1
\end{array}\right],
$$

and then, we have $P_{1}^{e}=\left(\tilde{x}_{1} / \tilde{d}_{1} \tilde{y}_{1} / \tilde{d}_{1} \tilde{z}_{1} / \tilde{d}_{1}\right)^{T}=\left(x_{1}\right.$ $\left.y_{1} z_{1}\right)^{T}$ to which we apply the rotation to obtain the $P_{2}^{e}$ point (see Figure $11 \mathrm{~b}$ ):

$$
P_{2}^{e}=\left(x_{2} y_{2} z_{2}\right)^{T}=R_{y}\left(\theta_{y}\right) \times R_{x}\left(\theta_{x}\right) \times P_{1}^{e},
$$

where $R_{y}\left(\theta_{y}\right)$ and $R_{x}\left(\theta_{x}\right)$ are rotation matrices around the $y$-axis and $x$-axis.

3. Finally, we express $P_{2}^{e}$ in $\mathcal{C S}_{i}$ to get the $P_{2}^{i}$ point (see Figure 11c):

$$
\left[\begin{array}{l}
\tilde{u}_{2} \\
\tilde{y}_{2} \\
\tilde{z}_{2} \\
\tilde{d}_{2}
\end{array}\right]=\left[\begin{array}{cccc}
1 & 0 & 0 & -w / 2 \\
0 & 1 & 0 & -w / 2 \\
0 & 0 & 1 & 0 \\
0 & 0 & 0 & 1
\end{array}\right]^{-1}\left[\begin{array}{c}
x_{2} \\
y_{2} \\
z_{2} \\
1
\end{array}\right] .
$$

We have $P_{2}^{i}=\left(\tilde{u}_{2} / \tilde{d}_{2} \tilde{v}_{2} / \tilde{d}_{2} \tilde{w}_{2} / \tilde{d}_{2}\right)^{T}=\left(\begin{array}{lll}u_{2} & v_{2} w_{2}\end{array}\right)^{T}$. The point we are looking for is $p_{2}^{i}=\left(u_{2} v_{2}\right)^{T}$. 


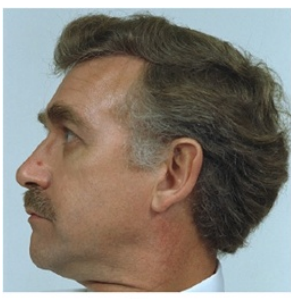

a

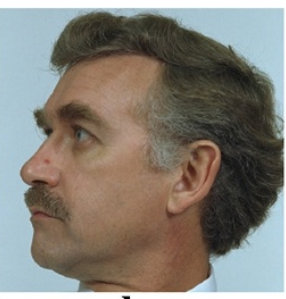

b

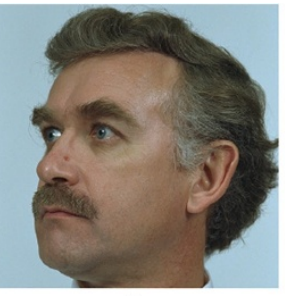

c

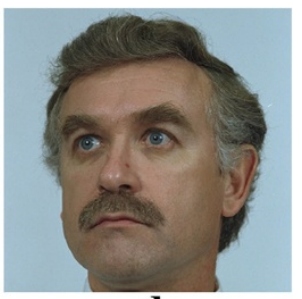

d

Figure 9 Example of faces with rotation-off-plane angles around the $\boldsymbol{y}$-axis. The face is turned $90^{\circ}$ in (a), $67.5^{\circ}$ in (b), $45^{\circ}$ in (c), and $22.5^{\circ}$ in (d)

To know the position of a subwindow $r_{i t}$ after a rotation, we apply the above process to the top left corner and to the bottom right corner of $r_{j t}$. The problem is that some subwindows can disappear (as shown in Figure 10c with the subwindow of $h_{3}$ in red). If a subwindow $r_{j t}$ disappears, then the associated weak classifier $h_{j t}$ becomes unavailable. By applying this rule to all the subwindows, the set of available weak classifiers can be defined and an associated McCascade can be built. Hence, creating a classifier that can detect non-upright faces calls for three steps:

1. Modifying the position of all subwindows using an ellipsoid model,

2. Defining the set of available weak classifiers by checking that their associated subwindows do not disappear after rotation, and

3. Creating the McCascade using available weak classifiers.

\subsection{A multiview system}

The solution presented in the last section aims to detect faces with a given ROP angle $\theta_{y}$. When faces with a ROP angle in a range $\left[-\theta_{y}^{\min },+\theta_{y}^{\max }\right]$ are to be detected, one solution is to combine several detectors. Each one is specialized in detecting faces with a given ROP angle $\theta_{y}$. In practice, it is generally assumed that each detector can detect faces in the range $\left[\theta_{y}-15, \theta_{y}+15\right]$. For example, if the total range is $[-45,+45]$, three detectors must be used: an upright face detector $H^{0}$, a detector of faces turned $+30^{\circ} H^{+30}$, and a detector of faces turned $-30^{\circ}$ $H^{-30}$. Detectors $H^{+30}$ and $H^{-30}$ are created by modifying all subwindow positions by $H^{0}$. To combine the three detectors, the solution proposed by Huang et al. [14] is applied. It is illustrated in Figure 12. To speed up the classification process, a pose estimator is used. For an input example $\mathbf{x}$, this estimation consists in applying the first three levels of every detector to $\mathbf{x}$. Then, the classification process continues with the detector that accepts $\mathbf{x}$ with the highest classification score. The pose estimation function is defined by:

$$
\operatorname{pose}(\mathbf{x})=\underset{\theta_{y} \in\{-30,0,30\}}{\operatorname{argmax}}\left(H_{3}^{\theta_{y}}(\mathbf{x})\right) .
$$

Note that the system used to combine the three detectors can be extended to get a face detector robust to pose and to occlusion. Indeed, using this system, several occlusion cascades (presented in Section 4.1) and several pose-specific detectors (presented in Section 5.1) can be combined.

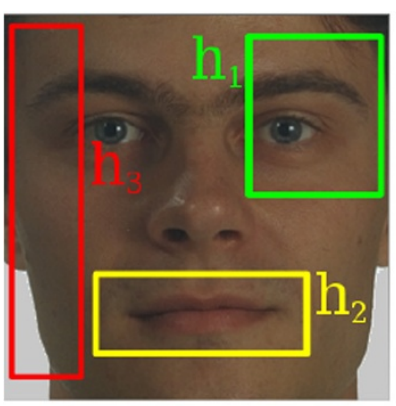

a

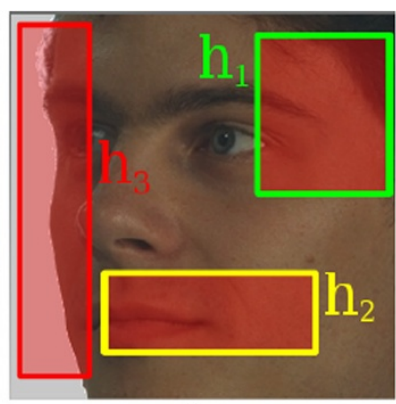

b

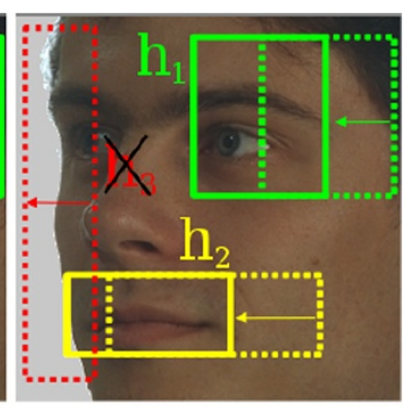

C

Figure 10 Detecting turned faces with an upright face detector. (a) An example of three discriminative subwindows of an upright face detector. $h_{1}, h_{2}$, and $h_{3}$ are the associated weak classifiers. (b) The face is turned $45^{\circ}$, and all subwindows could be classified as non-face. To alleviate the pose problem, we propose a three-dimensional geometric transformation to adjust all subwindow positions (see (c)). Note that the weak classifier $h_{3}$ becomes unavailable. 


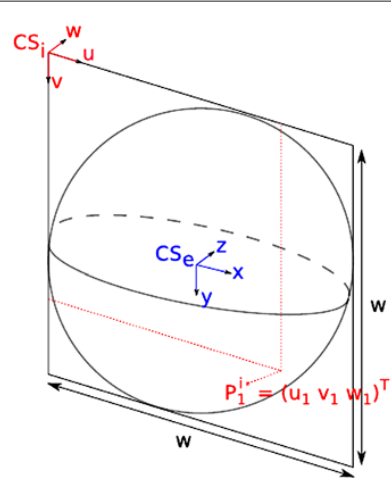

a

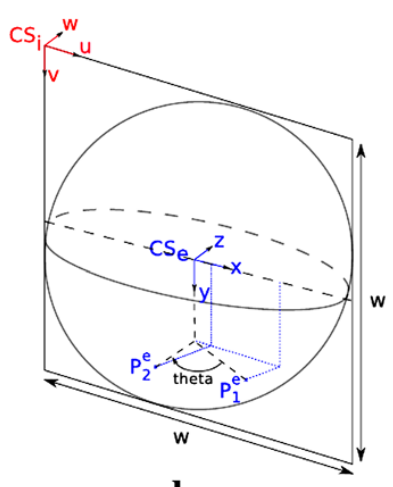

b

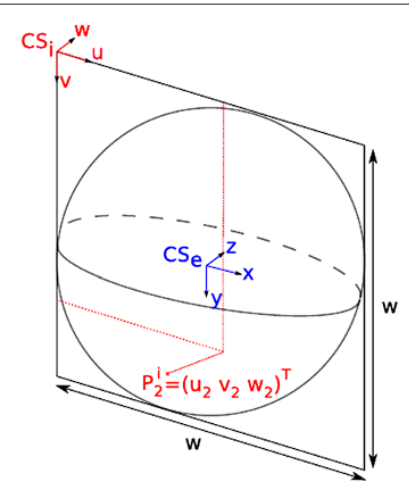

c

Figure 11 Rotation process of a point using an ellipsoid. (a) The image point $p_{1}^{i}=\left(u_{1}, v_{1}\right)$ is associated with the point $P_{1}^{i}=\left(u_{1}, v_{1}, w_{1}\right)$ on the ellipsoid using the ellipsoid equation. (b) $P_{1}^{i}$ is expressed in the ellipsoid coordinate system which gives the point $P_{1}^{e}$. The rotated point $P_{2}^{e}$ is computed using rotation matrices. (c) $P_{2}^{e}$ is expressed in the image coordinate system which gives the point $P_{2}^{i}=\left(u_{2}, v_{2}, w_{2}\right)$ and the image point $p_{2}^{i}=\left(u_{2}, v_{2}\right)$.

\section{Experiments}

This section presents the experiments achieved in order to (1) evaluate the performances of McCascade compared to the naive approach and (2) evaluate the McCascade algorithm for two concrete applications: occluded face detection and multiview face detection. In these experiments, upright face detectors are similar to the system of Tuzel et al. [15]: covariance matrices are used as features [16], and the learning algorithm is a cascade of LogitBoost [10]. Weak classifiers are linear functions that are learned from a set of feature vectors. A feature vector is derived from a covariance matrix by taking its upper triangular part. The only difference with the system [15] is that we assume that a feature vector lies on a vector space (in [15], a feature vector lies on a Riemannian manifold).

The first part of the experiments related to McCascade performance (Sections 6.2 and 6.3) are done with an upright face cascaded detector of three levels with 5, 10, and 25 weak classifiers, respectively. Positive examples come from the labeled upright faces in the wild database [17], and negative samples were generated from 1,310 images containing no face. A total of 4,000 positive examples and 8,000 negative examples are used to train each cascade level. The second part of the experiments related to applications (Sections 6.4, 6.5, and 6.6) are done with an upright face detector of nine levels. This detector is noted $\mathcal{C}$. Each level was trained with 5,000 positive examples and 5,000 negative examples. Each level was designed so that a detection rate of at least $d_{\min }=0.998$ and a false-positive rate of at most $f_{\max }=0.5$ were achieved on training examples. The positive examples again come from the labeled upright faces in the wild database, and negative samples were generated from 2,500 images containing no face. The FLANN library [18] is used to perform nearest neighbor searches (used in $P_{\mathrm{knn}}$ and $P_{\text {comb }}$ ). The test database is the CMU frontal face test A which consists of 42 images showing 169 upright faces with varied background [19].

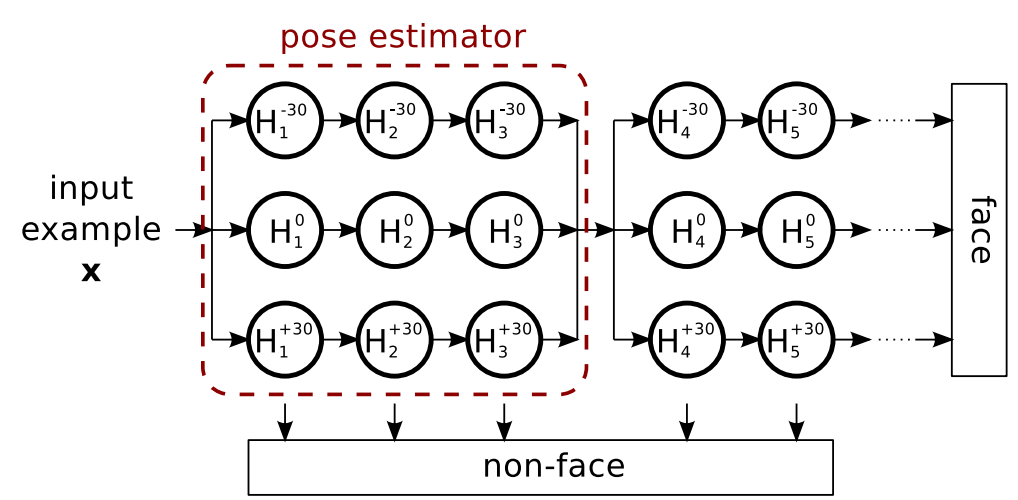

Figure 12 The multiview system. The input example $x$ first goes through the three levels of detectors $H^{-30}, H^{0}$, and $H^{+30}$. The estimated pose of $x$ is obtained by considering the detector that accepts $\mathrm{x}$ with the highest classification score, and then $\mathrm{x}$ continues with the selected detector. 
In the first part of the experiments, receiver operator characteristic (ROC) curves are used to evaluate and compare performances, and all performances exhibited are raw, i.e., the post-processing step of merging multiple detections is not taken into account here. This means that the false-positive rate can be reduced with this post-processing step without modifying the truepositive rate. When multiple detections occur for the same person, only the one with the highest classification score is kept. The others are simply ignored. In the second part of the experiments, free ROC(FROC) curves are used, and multiple detections are merged. Contrary to the ROC curve which plots detection rate versus false acceptance rate, the FROC curve plots the detection rate versus the number of false-positives and is more suited to evaluate performances of an object detector in specific applications. Different experiments were conducted to evaluate the different aspects of our method. In Section 6.2, we test the three proposed cost functions TP_cost, FP_cost, and FP_TP_cost used in the computation of McCascade's thresholds. Then, Section 6.3 deals with the evaluation of the different strategies used to estimate posterior probability: $P_{\text {boost }}, P_{\text {knn }}$, and $P_{\text {comb. }}$. After these two series of experiments, we apply our method to two specific applications: detecting faces occluded by a scarf or sunglasses (see Section 6.4) and detecting faces in a pose different than the one learned (see Section 6.5).

\subsection{Good detection criterion}

Building ROC or FROC curves requires computing truepositive rates and false-positive rates. A criterion must be defined to decide if a given detection is a true-positive or a false-positive. The criterion used in these experiments is defined in the overlap between the detection and the ground truth. It was proposed by Yao and Odobez [20]. The overlap is computed with the $F$ measure $F_{\text {overlap: }}$ :

$$
\begin{array}{r}
F_{\text {overlap }}(\mathrm{GT}, D)=\frac{2 \rho \pi}{\rho+\pi} \quad \text { where } \quad \rho=\frac{|\mathrm{GT} \cap D|}{|\mathrm{GT}|} \\
\text { and } \pi=\frac{|\mathrm{GT} \cap D|}{|D|} .
\end{array}
$$

$\rho$ stands for the precision area and $\pi$ for the recall area. GT is the ground truth area, and $D$ is the detection area. The operator $|R|$ is the number of pixels in the area $R$. A detection matches with ground truth if $F_{\text {overlap }}>0.5$.

\subsection{Evaluation of threshold estimation strategies}

In this first part, we evaluate the influence of the cost function in threshold $\beta_{j}$ estimation when a given proportion

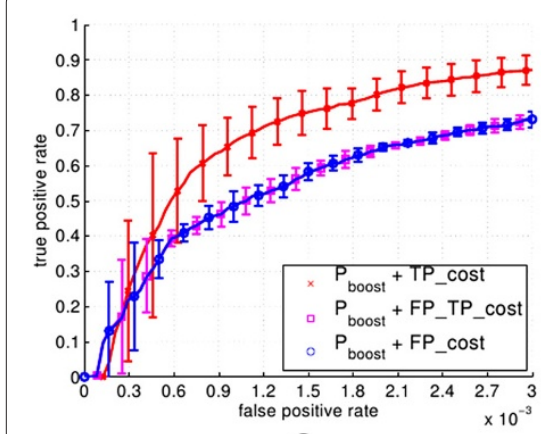

$\mathbf{a}$

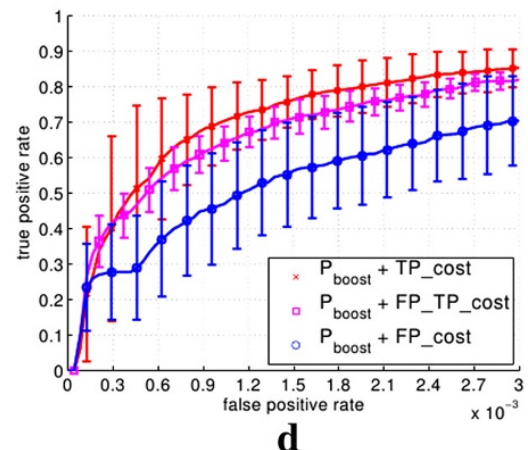

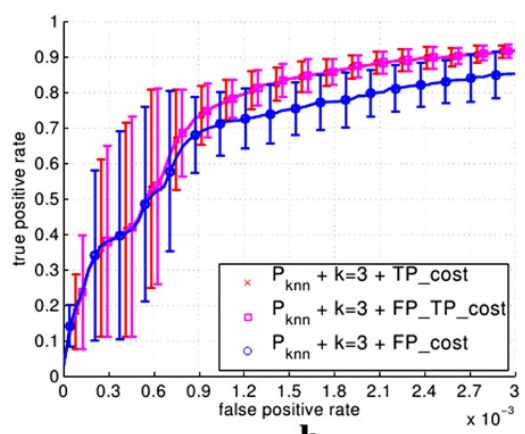

b

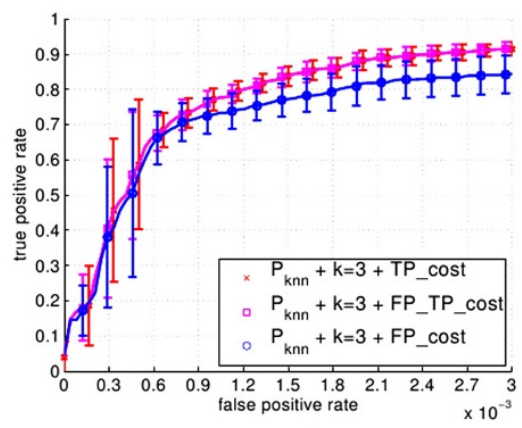

e

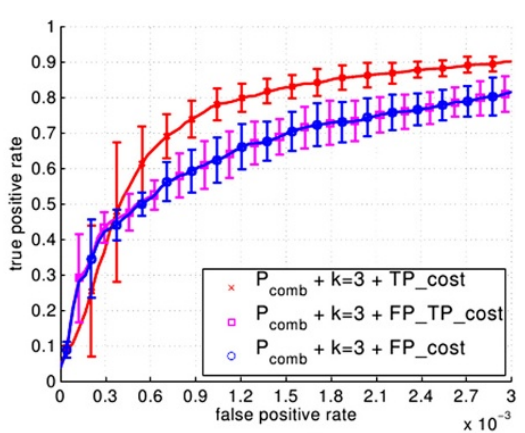

C

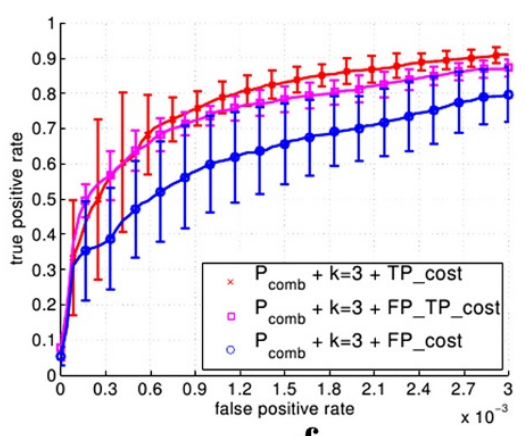

f

Figure 13 Performance of classifiers produced with the three cost functions: TP_cost, FP_cost and FP_TP_cost. In (a, b, c), 50\% of the weak classifiers are unavailable, while $60 \%$ of the weak classifiers are unavailable in $(\mathbf{d}, \mathbf{e}, \mathbf{f})$. In (a) and (d), posterior probabilities are computed with $P_{\text {boost }}$. In (b) and (e), $P_{\mathrm{knn}}$ is used, and $P_{\text {comb }}$ is used in (c) and (f). The number of neighbors in $P_{\mathrm{knn}}$ and $P_{\text {comb }}$ is fixed at 3. 
of weak classifiers is missing. We chose to consider $50 \%$ and $60 \%$ of missing weak classifiers because these rates are realistic in occluded face detection. Given a missing weak classifier rate, we randomly create two sets of weak classifiers per level to be considered as unavailable. For example, consider the level 2 of the classifier which has ten weak classifiers. If $60 \%$ of the weak classifiers are missing, then 6 weak classifiers must be selected as unavailable. For each of the two sets of unavailable weak classifiers, we randomly select six weak classifiers to be considered as unavailable. These two sets could be $\left\{h_{21}, h_{22}, h_{23}, h_{24}\right.$, $\left.h_{27}, h_{29}\right\}$ and $\left\{h_{22}, h_{23}, h_{25}, h_{26}, h_{27}, h_{28}\right\}$. Given the sets of the three levels, there are $2 \times 2 \times 2=8$ possible configurations to test resulting in eight ROC curves. Means and standard deviations are then computed to produce the final ROC curve. For each configuration, thresholds are first computed and the resulting classifier is applied to the test database. This test process is repeated for each cost function associated to each posterior probability computation strategy: $P_{\text {boost }}, P_{\mathrm{knn}}$, and $P_{\text {comb }}$. For the last two strategies, we fix the number of neighbors $k$ at 3 . All the ROC curves are available in Figure 13. In all the curves, the cost function TP_cost produces a classifier that outperforms the other classifiers produced with FP_cost and FP_TP_cost.

ROC curves are useful in evaluating the overall performance of a classifier. When we train a classifier, this presents a given true-positive rate and a given falsepositive rate which should be consistent with the application targeted. In face detection, we are interested in having a high true-positive rate and a low false-positive rate. This is why, in addition to ROC curves, we present the false-positive rate, noted FP, and the true-positive rate, noted TP, of classifiers produced by the three cost functions. Results for a missing rate of $50 \%$ can be found in Table 1, while results for $60 \%$ are available in Table 2 . In these tables, we also print the mean number of levels evaluated per negative example, noted $\overline{h_{\text {level }}}$. This criterion reflects the impact of the cost function on the execution time of the classifier. Indeed, a high number of evaluated levels per negative example will bring a high execution time. In both tables, we print in italics the cost function that provides the most consistent performance. As expected, the use of cost functions FP_cost and FP_TP_cost involves low false-positive rates but also involves low true-positive rates (some of them lower than $10 \%$ ), which means that these classifiers do not have a practical value. Furthermore, the impact on the mean number of evaluated levels is not very significant: we note an increase of about 7\% between the cost function TP_cost and the two others. These experiments prompt us to keep the cost function TP_cost because FP_cost and FP_TP_cost tend to decrease the true-positive rate and the overall performance.
Table 1 Evaluation of cost function used to compute thresholds $\beta_{j}$ when $\mathbf{5 0 \%}$ of weak classifiers are missing

\begin{tabular}{|c|c|c|c|c|c|}
\hline & $k$ & Cost function & $\begin{array}{c}\text { FP } \\
\times 10^{-3}\end{array}$ & TP & $\overline{n_{\text {level }}}$ \\
\hline & & $T P_{-}$cost & 3.21 & 0.88 & 1.61 \\
\hline \multirow[t]{3}{*}{$P_{\text {boost }}$} & - & FP_cost & 0.066 & 0.1 & 1.48 \\
\hline & & FP_TP_cost & 0.08 & 0.15 & 1.48 \\
\hline & & TP_cost & 5.56 & 0.95 & 1.29 \\
\hline \multirow[t]{3}{*}{$P_{k n n}$} & 3 & FP_cost & 0.14 & 0.52 & 1.26 \\
\hline & & FP_TP_cost & 0.17 & 0.44 & 1.29 \\
\hline & & $T P_{-}$cost & 5.43 & 0.95 & 1.62 \\
\hline \multirow[t]{2}{*}{$P_{\text {comb }}$} & 3 & FP_cost & 0.006 & 0.12 & 1.48 \\
\hline & & FP_TP_cost & 0.03 & 0.24 & 1.48 \\
\hline
\end{tabular}

Three evaluation terms are exposed: the false positive rate, the true positive rate and the mean number of evaluated levels per negative example noted $\overline{n_{\text {level }}}$.

\subsection{Performance of the posterior probability estimation}

In this section, we evaluate the three strategies to estimate posterior probabilities proposed in Section 3.3: $P_{\text {boost }}$, $P_{\text {knn }}$, and $P_{\text {comb }}$. The evaluation methodology is the same as the previous section (same cascaded detector, same test database, same missing rate). Here, the cost function used to compute thresholds is TP_cost. Five configurations are compared: (1) 'CascadeF' is the initial cascade with the full set of weak classifiers (can be seen as an upper bound), (2) 'CascadeA' is the naive approach presented in Section 3.1 where the initial cascade is only used with available weak classifiers, (3) 'McCascade + Pboost' is a McCascade used with available weak classifiers where posterior probabilities are computed with $P_{\text {boost }}$, (4) 'McCascade + Pknn' is a McCascade used with available weak classifiers where posterior probabilities are computed with $P_{\text {knn }}$, and (5) 'McCascade + Pcomb' is

Table 2 Evaluation of cost function used to compute thresholds $\beta_{j}$ when $60 \%$ of weak classifiers are missing

\begin{tabular}{|c|c|c|c|c|c|}
\hline & $k$ & Cost function & $\begin{array}{c}\mathrm{FP} \\
\times 10^{-3}\end{array}$ & $\mathrm{TP}$ & $\overline{n_{\text {level }}}$ \\
\hline \multirow{3}{*}{$P_{\text {boost }}$} & \multirow{3}{*}{-} & $T P_{-}$cost & 8.4 & 0.95 & 1.64 \\
\hline & & FP_cost & 0.058 & 0.06 & 1.48 \\
\hline & & FP_TP_cost & 0.17 & 0.29 & 1.49 \\
\hline \multirow{3}{*}{$P_{k n n}$} & \multirow{3}{*}{3} & $T P_{-}$cost & 8.25 & 0.96 & 1.32 \\
\hline & & FP_cost & 0.15 & 0.56 & 1.26 \\
\hline & & FP_TP_cost & 0.28 & 0.58 & 1.32 \\
\hline \multirow{3}{*}{$P_{c o m b}$} & \multirow{3}{*}{3} & TP_cost & 11.9 & 0.97 & 1.67 \\
\hline & & FP_cost & 0.005 & 0.11 & 1.48 \\
\hline & & FP_TP_cost & 0.16 & 0.49 & 1.5 \\
\hline
\end{tabular}

Three evaluation terms are exposed: the false positive rate, the true positive rate and the mean number of evaluated levels per negative example noted $\overline{n_{\text {level }}}$. 
a McCascade used with available weak classifiers where posterior probabilities are computed with $P_{\text {comb. }}$. When $P_{\text {knn }}$ and $P_{\text {comb }}$ are used, only the best results are plotted $\left(k=7\right.$ for $P_{\text {knn }}$ and $k=3$ for $\left.P_{\text {comb }}\right)$. The results can be found in Figure 14. In both cases, the McCascade structure improves the performance. The most interesting results are obtained when $P_{\text {knn }}$ and $P_{\text {comb }}$ are used. In that case, the true positive rate increases from 10 to $30 \%$ when $50 \%$ of weak classifiers are unavailable. When $60 \%$ of weak classifiers are unavailable, the improvement is even higher: from $20 \%$ to $60 \%$. In both cases, the proposed method outperforms the naive approach. Moreover, McCascade is really more stable than the naive approach (see standard deviations in each curve) which ensures good performance in every case. Finally, the proposed method does not suffer from the additional $10 \%$ of unavailable weak classifiers. Even if $P_{\mathrm{knn}}$ and $P_{\text {comb }}$ are close in terms of performance, we note that $P_{\mathrm{knn}}$ is slightly better.

The influence of the number of neighbors in the McCascade coupled with the strategy $P_{\mathrm{knn}}$ can be found in Figure 15. In both cases, $k=7$ gets the best performances, but $k=3$ should be preferred as it provides similar performance and lower computational cost. In all the following experiments, the McCascade is used with the $P_{\mathrm{knn}}$ strategy and $k=3$.

An additional result is given in the Figure 16 where $30 \%$ of the weak classifiers are missing. Below this rate of $30 \%$, the naive approach and the McCascade get close performances. However, when at least $30 \%$ of the weak classifiers are missing, using a McCascade becomes interesting. Indeed, it can be noted in Figure 16 that a McCascade with the strategy $P_{\mathrm{knn}}$ increases the true-positive rate up to $30 \%$ compared to the naive approach.

\subsection{Occluded face detection}

In this section, we evaluate the performance of McCascade coupled with the principle of cascading with evidence in a specific application: detecting faces with top occlusions (like sunglasses) or bottom occlusions (like a scarf). We only consider these two types of occlusions for two reasons. The first is that we are working in a video surveillance context in which these two types of occlusions are often encountered. The second reason is that a public database with these two types of occlusion is available: the AR database.

\subsubsection{Evaluation on the AR database}

The AR database [21] is used first. In particular, we use the 765 images of faces occluded by a scarf and the 765 images of faces occluded by sunglasses. The classifier used here is the upright face detector of nine levels. Using this cascade $\mathcal{C}$, we build a McCascade $\mathcal{C}^{\mathcal{A}}$ that can handle bottom occlusion and a McCascade $\mathcal{C}^{\mathcal{B}}$ that can handle top occlusion. Also, a detector that associates $\mathcal{C}, \mathcal{C}^{\mathcal{A}}$, and $\mathcal{C}^{\mathcal{B}}$ with the principle of cascading with evidence is created. This detector will be noted 'McCascades + evidence' in the results. The $\mathrm{McCascade} \mathcal{C}^{\mathcal{A}}$ has, on average, $42 \%$ unavailable weak classifiers per level. The McCascade $\mathcal{C}^{\mathcal{B}}$ has, on average, $46 \%$ unavailable weak classifiers per level.

Two scenarios are tested:

- Scenario 1. We consider images of faces occluded by a scarf, and we then compare (1) the cascade $\mathcal{C}$, (2) the McCascade $\mathcal{C}^{\mathcal{A}}$, and (3) the detector McCascades + evidence.

- Scenario 2. We consider images of faces occluded by sunglasses, and we then compare (1) the cascade $\mathcal{C}, 2$ ) the McCascade $\mathcal{C}^{\mathcal{B}}$, and (3) the detector McCascades + evidence.

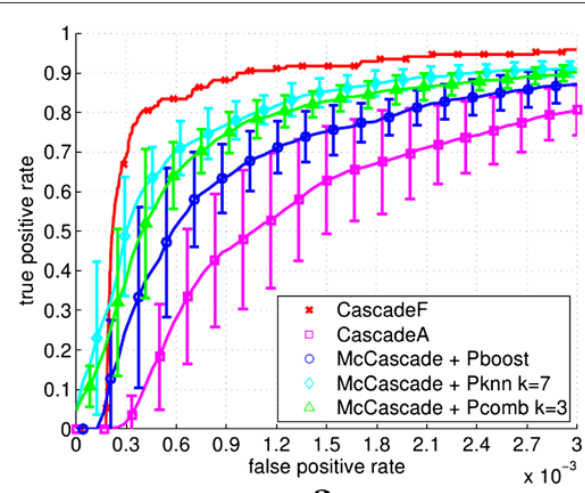

a

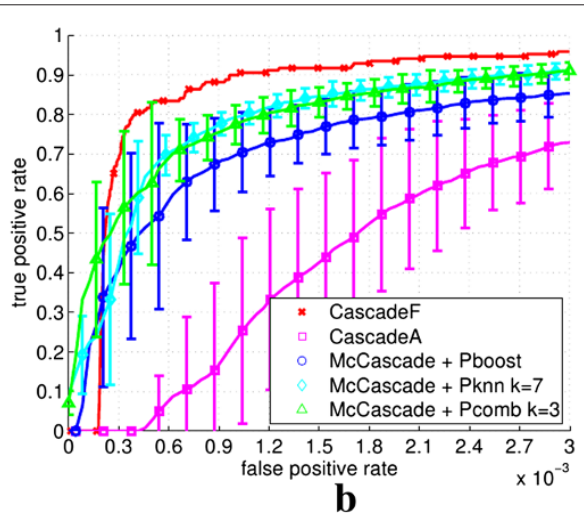

b

Figure 14 Comparison of different strategies to estimate posterior probability in a boosted McCascade. They are for different rates of missing weak classifiers. In (a), 50\% of weak classifiers are missing, while in (b), 60\% are missing. Each McCascade can be compared with the naive approach presented in Section 3.1 where the initial boosted cascade is used with available weak classifiers (noted CascadeA). In each curve, we also plot the performance of the boosted cascade when all weak classifiers are known (noted CascadeF) to show the effect of missing weak classifiers on initial performance (best view in color). 


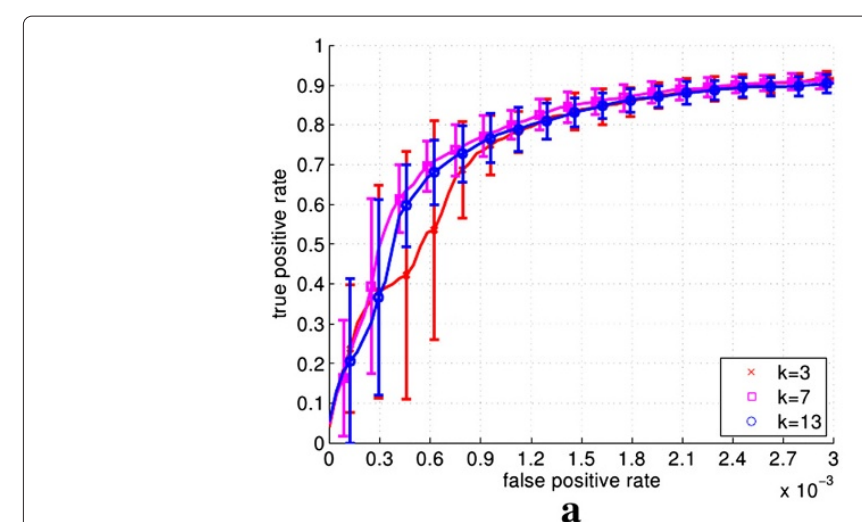

Figure 15 Influence of the number of nearest neighbors $k$ in the strategy $P_{\mathrm{knn}}$ weak classifiers are missing.

For all scenarios, FROC curves are computed. To create the FROC curve of a cascaded detector, several threshold values are tested for the last level which results in corresponding points of detection rate and number of false-positives. To get more points (points with a higher detection rate and a higher number of false-positives), the last level must be removed, and then different thresholds for the new last level are tested. This procedure continues until enough points are collected. When several cascades are associated (e.g., in the system ${ }^{'} \mathcal{C}+\mathcal{C}^{\mathcal{A}}+\mathcal{C}^{\mathcal{B}}+$ evidence'), creating a FROC curve is not straightforward because each cascade has its own thresholds. To alleviate this problem, we use the idea proposed by Viola and Jones in [22]. To create FROC curves from multiple cascades, thresholds are simultaneously modified in all cascades. In the same way, layers are simultaneously removed in all cascades.

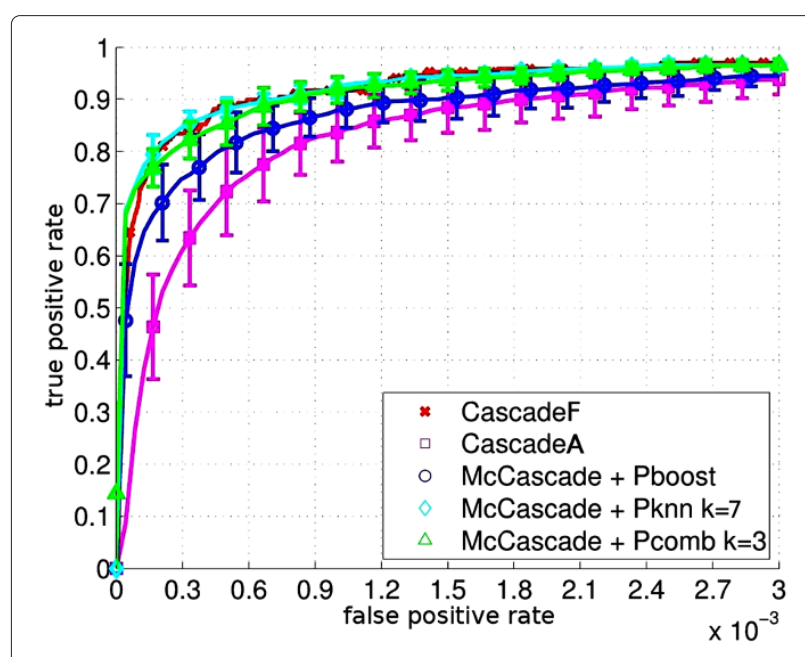

Figure 16 A McCascade becomes interesting when at least 30\% of the weak classifiers are missing.

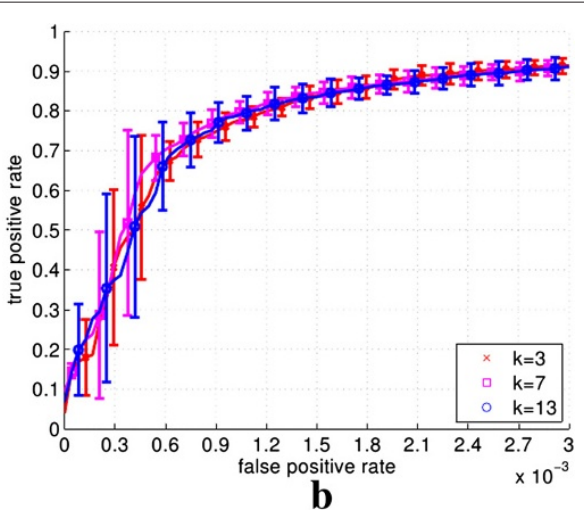

(a), $50 \%$ of weak classifiers are missing, and in (b), $60 \%$ of
The FROC curve of scenario 1 is available in Figure 17. The McCascade $\mathcal{C}^{\mathcal{A}}$ (noted 'McCascade') greatly improves the detection rate (up to $30 \%$ ). The drawback of $\mathcal{C}^{\mathcal{A}}$ is that it is designed to detect faces with bottom occlusions. When the encountered occlusion is unknown (top or bottom), the detector McCascades + evidence can be used, and Figure 17 shows that its performances are close to the ones of $\mathcal{C}^{\mathcal{A}}$.

The FROC curve of scenario 2 is available in Figure 18a. On faces occluded by sunglasses, the initial cascade and the proposed solutions (the detector $\mathcal{C}^{\mathcal{B}}$ and the detector McCascades + evidence) expose very poor results. The poor results in scenario 2 are due to a limitation in our solution: the fact that each weak classifier does not have the same performance. Several works on face detection noticed that learned weak classifiers often rely on the

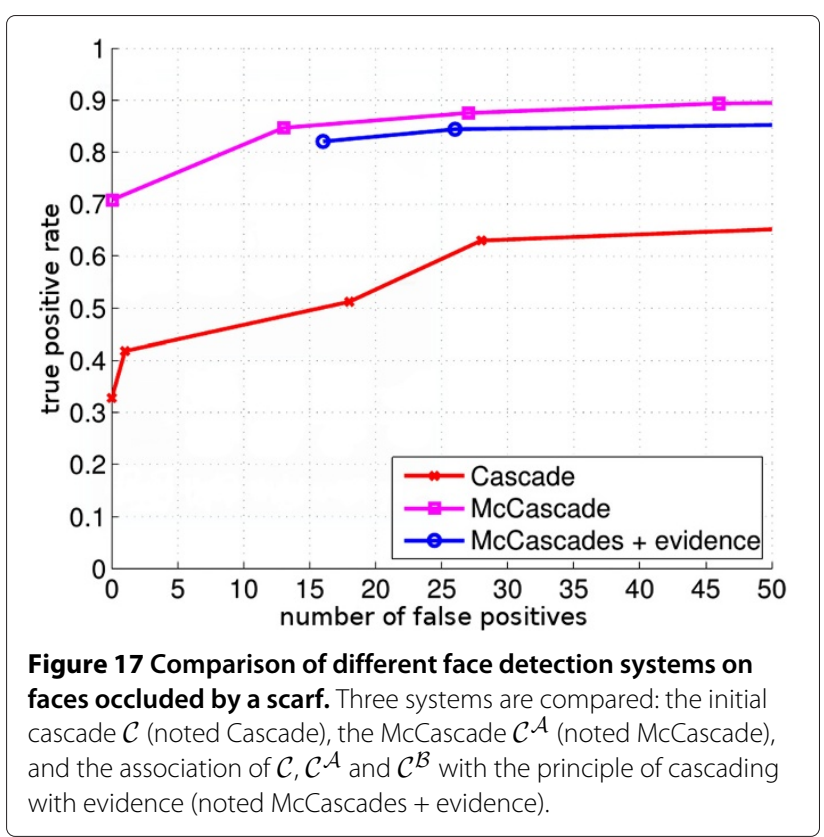




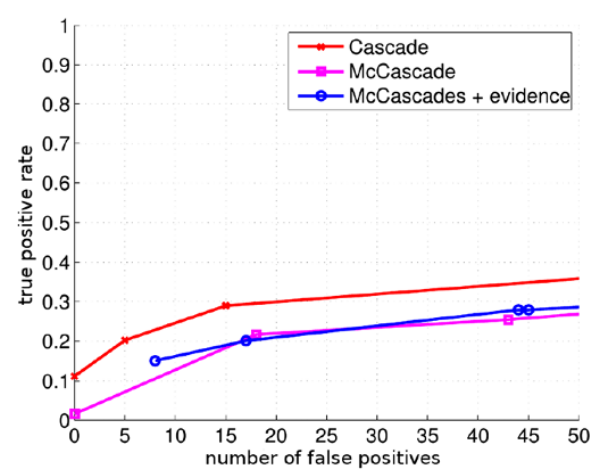

$\mathbf{a}$

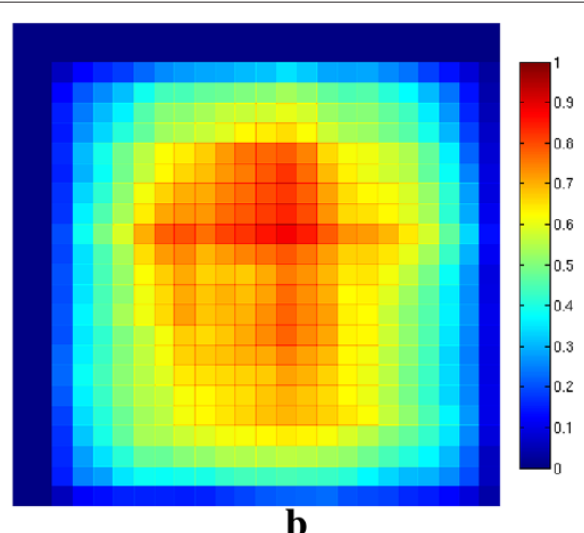

b

Figure 18 Limitation of the proposed solution. (a) Comparison of the initial cascade $\mathcal{C}$ (noted Cascade), the McCascade $\mathcal{C}^{\mathcal{B}}$ (noted McCascade), and the association of $\mathcal{C}, \mathcal{C}^{\mathcal{A}}$, and $\mathcal{C}^{\mathcal{B}}$ with the principle of cascading with evidence (noted McCascades + evidence) on faces occluded by sunglasses. (b) Performance map of all the weak classifiers in the initial cascade. Note that most of the performance is located on the upper part of the face (best seen in color).

upper part of the face to make a decision because the eye area is very discriminative. When our upright face detector was trained, we noticed the same phenomenon: most of the weak classifiers are located on the upper part of the face, and they are more powerful than the weak classifiers located on the lower part of the face. This fact can be seen in Figure $18 \mathrm{~b}$ which represents a performance map $\mathcal{M}$ of all the weak classifiers in the initial cascade. To build this map, we first initialize all values to zero. Then, for all the weak classifier $h_{j t}$, we compute its classification rate $C R_{j t}$ (rate of well-classified positive and negative examples), and we update $\mathcal{M}$ with:

$$
\mathcal{M}(x, y)=\mathcal{M}(x, y)+C R_{j t} \quad \forall(x, y) \in \mathcal{S}_{j t} \subset \mathcal{M} .
$$

Finally, we normalize all the values between 0 and 1 . Based on this map, we understand that our method fails on faces occluded by sunglasses because, in this scenario, we only use weak classifiers located on the lower part of the face which are too weak to ensure good performance.

In scenario 2, the existing solutions such as [7] will exhibit better results. Indeed, a specific classifier will be trained to detect faces with top occlusions. In scenario 1 , it is interesting to compare our system with [7]. Rather than building the complete system described in [7], a specific classifier was trained to detect faces with bottom occlusion. This specific classifier is close to cascade $\mathcal{C}$, except that all the learned weak classifiers are located on the area that it is not occluded. This specific classifier is then compared with the McCascade $\mathcal{C}^{\mathcal{A}}$. Results can be found in the Figure 19. Except with a very low number of falsepositives, the specific classifier gets a higher detection rate (up to $10 \%$ ).

\subsubsection{Evaluation in real-life scenario}

A test is also done in a real-life scenario. A camera is placed on a pole to film a group of 15 persons. Some of them have their face occluded by a scarf, coat, or hood. Examples of images from the sequence are available in Figure 20. There is a small angle (around $20^{\circ}$ ) between the optical axis of the camera and the ground to imitate conditions of a video surveillance context.

Three detectors are applied to this sequence:

- Upright face detector $\mathcal{C}$. It is noted ' $F D_{\text {cov }}$ ' in the results.

- Detector that associates $\mathcal{C}, \mathcal{C}^{\mathcal{A}}$, and $\mathcal{C}^{\mathcal{B}}$ with the principle of cascading with evidence. It is noted ' $\mathrm{FD}_{\mathrm{cov}}+$ occlusion' in the results.

- Upright face detector of the OpenCV library (the file

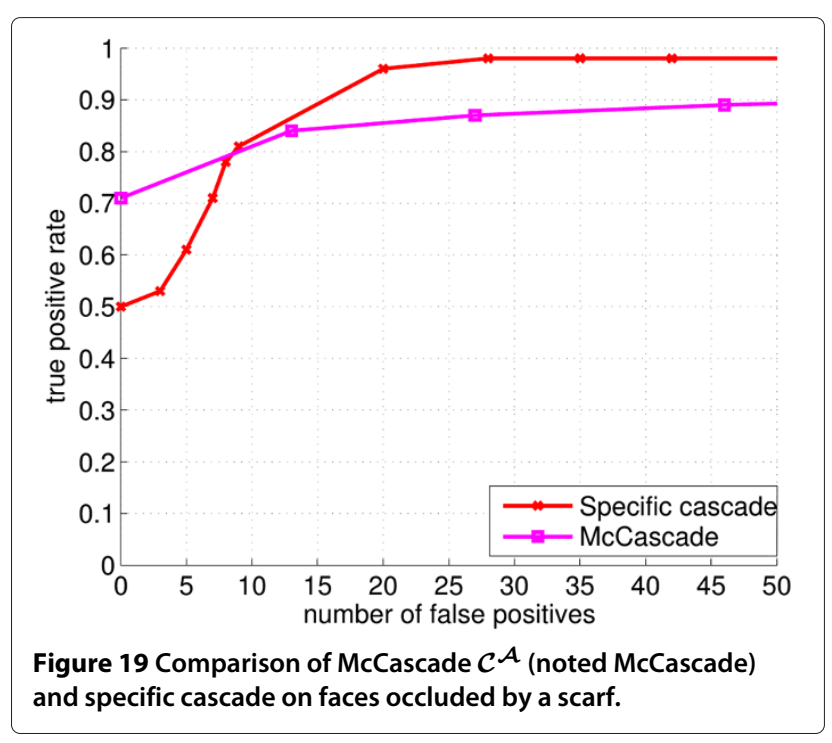




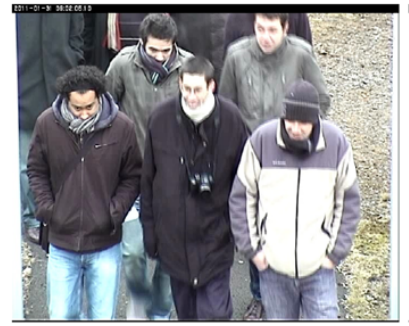

a

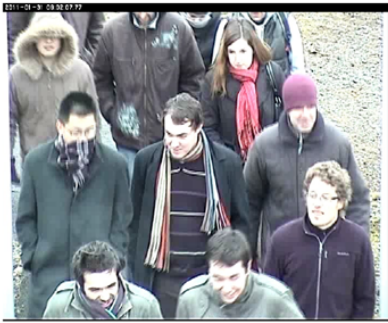

b

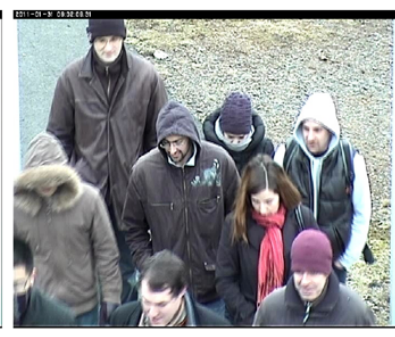

c

Figure $\mathbf{2 0}$ Images from a realistic sequence. A group of 15 persons are filmed by a camera on a pole. Some of them have their face occluded by a scarf, coat, or hood. The 15 persons can be seen in (a), (b), and (c).

haarcascade_frontalface_alt_tree.xml is used). This detector is the implementation of the solution of Lienhart et al. [23]. This classifier is a cascade of boosted classifiers. Haar features are used. It is noted ' $F D_{\text {haar }}$ ' in the results.

The detector $\mathrm{FD}_{\text {haar }}$ just gives output detections. The classification score of each detection is not known. This detector is applied first on the sequence. Then, with the help of ground truth, the detection rate per person is computed. The number of false-positives nbFP $_{\text {haar }}$ is also noted. The other two detectors are then applied to the sequence. The rejection thresholds of the two detectors are modified so that they obtain nbFP haar false-positives. Then, the detection rate per person is computed. The results are available in Figure 21. The red line is the average detection rate of the detector $\mathrm{FD}_{\text {haar }}$. The yellow line is the average detection rate of the detector $\mathrm{FD}_{\mathrm{cov}}$, and

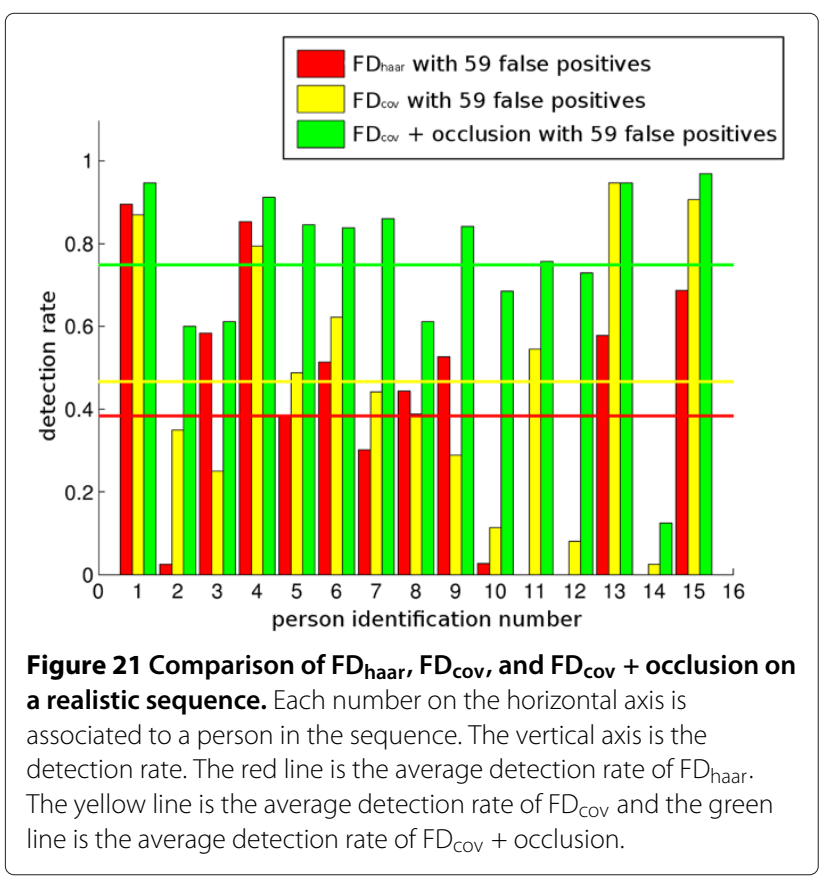

the green line is the average detection rate of the detector $\mathrm{FD}_{\text {cov }}+$ occlusion. The worst performances are obtained with $\mathrm{FD}_{\text {haar }}$ with $38 \%$ true-positive rate. $\mathrm{FD}_{\text {cov }}$ gets a $47 \%$ true-positive rate. The best performances are achieved by $\mathrm{FD}_{\mathrm{cov}}+$ occlusion with a true positive rate of $75 \%$. Moreover, we note that $\mathrm{FD}_{\text {haar }}$ does not detect persons 11,12 , and 14 . They are detected by the other two classifiers. Detection examples of these persons are given in Figure 22.

\subsection{Multiview face detection}

In this part of the experiments, the boosted McCascade algorithm has been applied to another specific application: detecting faces in different poses using an upright face detector. The FERET database [24] was used to evaluate the system. We test our method on faces turned $22.5^{\circ}, 45^{\circ}$ and $67.5^{\circ}$. For each angle, all the subwindow positions are first adjusted using the procedure described in Section 5.

\subsubsection{Ellipsoid parameters}

To modify the subwindow positions, parameters $w, a, b$, and $c$ must be fixed. Parameter $w$ corresponds to the size of the training images which is 24 in our case. To fix ellipsoid parameters $a, b$, and $c$, we do an exhaustive search and keep the parameters, giving the best results on validation sets from the FERET database. Two validation sets were created: one for the angle $22.5^{\circ}$ and one for $45^{\circ}$. For each angle, we keep half of the images to fix the ellipsoid parameters. The other half is used to evaluate the complete system. For each parameter value $\left(a_{i}, b_{i}, c_{i}\right)$, we apply the following methodology:

1. Based on the upright face classifier, we create two classifiers $\mathcal{C}^{22.5}$ and $\mathcal{C}^{45}$ by adjusting all the subwindow positions using ellipsoid parameters $\left(a_{i}, b_{i}, c_{i}\right)$. Subwindows that disappear are handled by the naive approach presented in Section 3.1, i.e., associated weak classifiers are simply ignored.

2. $\mathcal{C}^{22.5}$ is applied to the validation set of images of faces turned $22.5^{\circ}$, and the ROC curve is computed. Then, 


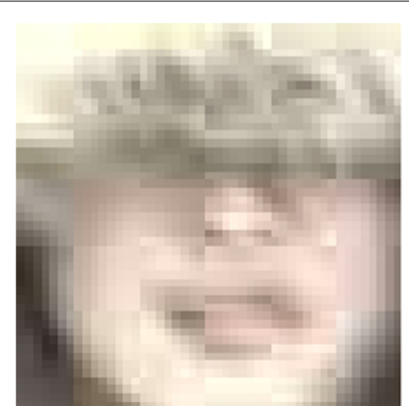

$\mathbf{a}$

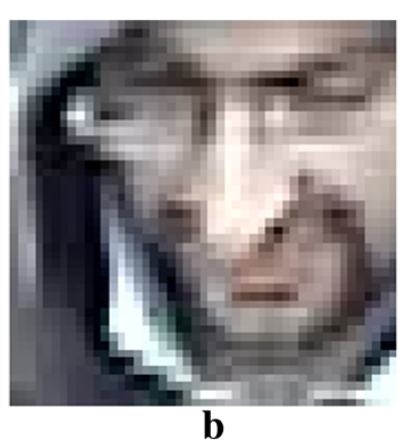

Figure 22 Persons that are not detected by $\mathrm{FD}_{\text {haar }}$. In (a), the person is occluded by a hood. In (b), the glasses and the beard make the person

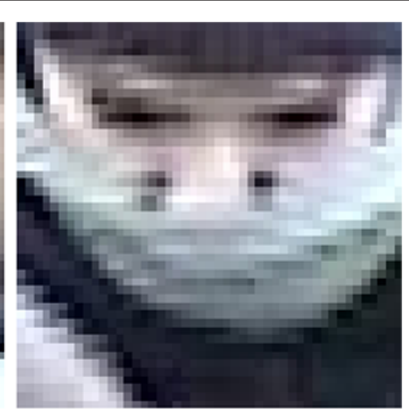

c difficult to detect. In (c), the person is occluded by a scarf.

the area under ROC curve is computed which gives $\operatorname{auc}_{i}^{22.5}$ (auc is a criterion to compare ROC curves:

the higher it is, the better the ROC curve). Using $\mathcal{C}^{45}$, we also get auc ${ }_{i}^{45}$.

3. Finally, the overall value $\operatorname{auc}_{i}=\operatorname{auc}_{i}^{22.5}+\operatorname{auc}_{i}^{45}$ is computed.

Parameters with the best value $\mathrm{auc}_{i}$ were kept. We found that $a=2.0 * w / 2, b=w$. and $c=w / 2$ give the best results.

\subsubsection{Modification of subwindow positions}

Here, the use of an ellipsoid to modify subwindow positions is evaluated. Three detectors are built:

- $\mathcal{C}^{22.5}$ is a detector of faces that turned $22.5^{\circ}$,

- $\mathcal{C}^{45}$ is a detector of faces that turned $45^{\circ}$, and

- $\mathcal{C}^{67.5}$ is a detector of faces that turned $67.5^{\circ}$.

Each one is built from $\mathcal{C}$ by modifying subwindow positions. Subwindows that disappear are handled by the naive approach. These detectors are then applied to images from the FERET database. The results are available in Figures 23 and 24. In each curve, the upright face detector $\mathcal{C}$ is noted 'Cascade.' Detectors $\mathcal{C}^{22.5}, \mathcal{C}^{45}$ and $\mathcal{C}^{67.5}$ are noted 'MaCascade' (for cascade with multiview adaptation). On faces turned $22.5^{\circ}$, the improvement is slight because the appearance of such faces is still close to the appearance of upright faces. The improvement is greater on faces turned $45^{\circ}$. Indeed, the detection rate increases from $30 \%$ to $40 \%$. Finally, we see that the detection of faces turned $67.5^{\circ}$ can be seen as a limitation of the proposed method. A detection rate increase (up to $60 \%$ ) only occurs when the number of false-positives becomes high (>30). This limitation comes from the step of adjusting the subwindow positions:

1. The subwindow position modification should compensate the modified appearance of a turned face of an angle $\theta_{y}$. When the angle $\theta_{y}$ increases, it becomes much more difficult to compensate the modified appearance as the modification becomes stronger and stronger.

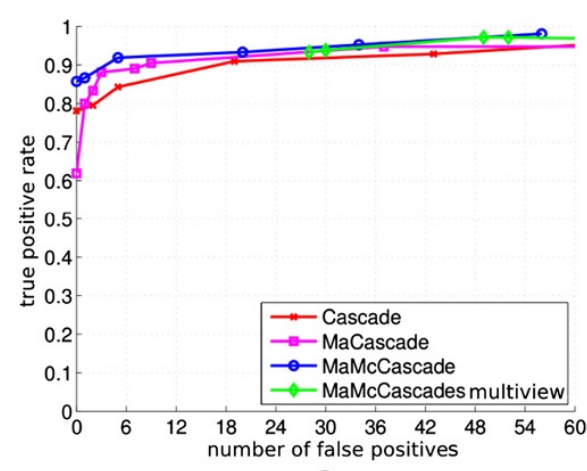

a

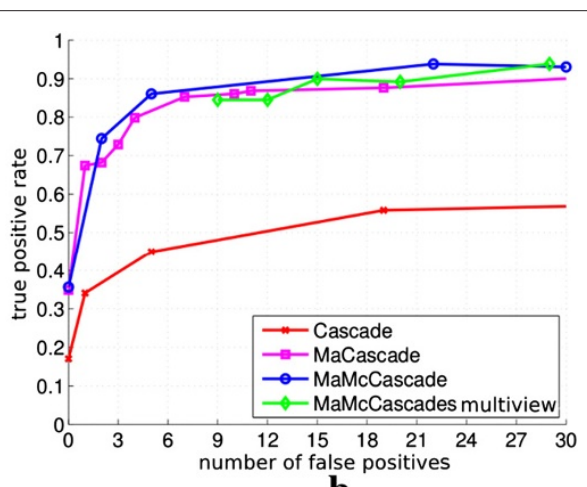

b

Figure 23 Performances of different detectors on faces turned $22.5^{\circ}$ in (a) and $\mathbf{4 5}$ in (b). The detector Cascade is the upright face detector. The detector MaCascade is built from the upright face detector and aims to detect turned faces. Subwindow positions are modified and unavailable weak classifiers are handled by the naive approach. The detector MaMcCascade is the same detector as MaCascade except that unavailable weak classifiers are handled with a McCascade. The detector MaMcCascade multiview is a multiview system that combines three MaMcCascades: one for the angle $22.5^{\circ}$, one for $45^{\circ}$, and one for $67.5^{\circ}$. 


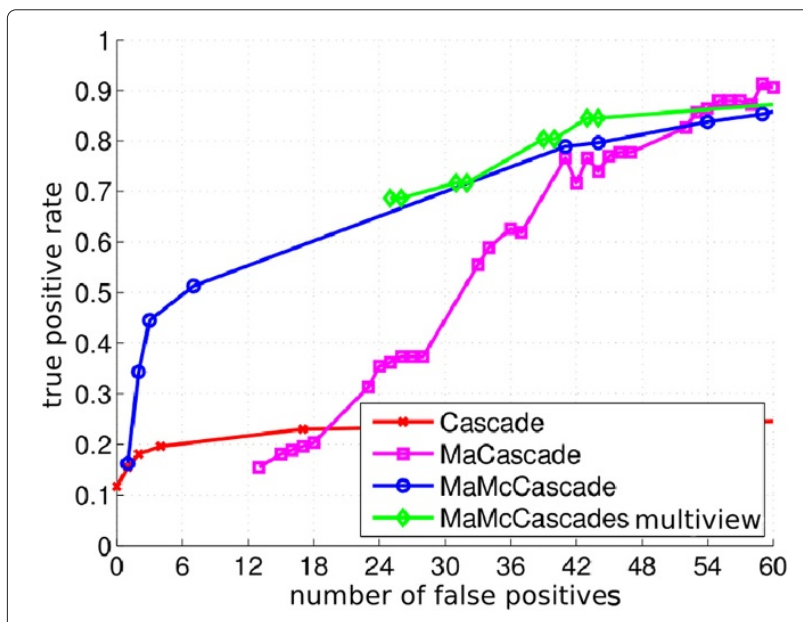

Figure 24 Performances of different detectors on faces turned $\mathbf{6 7 . 5}^{\circ}$. The detector Cascade is the upright face detector. The detector MaCascade is built from the upright face detector and aims to detect turned faces. Subwindow positions are modified and unavailable weak classifiers are handled by the naive approach. The detector MaMcCascade is the same detector as MaCascade except that unavailable weak classifiers are handled with a McCascade. The detector MaMcCascade multiview is a multiview system that combines three MaMcCascades: one for the angle $22.5^{\circ}$, one for $45^{\circ}$, and one for $67.5^{\circ}$.

2. In Section 5, we explain that some subwindows can disappear due to rotation. In fact, the number of subwindows that disappear increases with the angle $\theta_{y}$. This loss impacts the initial performance.

\subsubsection{Association with a McCascade}

The three detectors of the previous section $\mathcal{C}^{22.5}, \mathcal{C}^{45}$, and $\mathcal{C}^{67.5}$ have some unavailable weak classifiers:

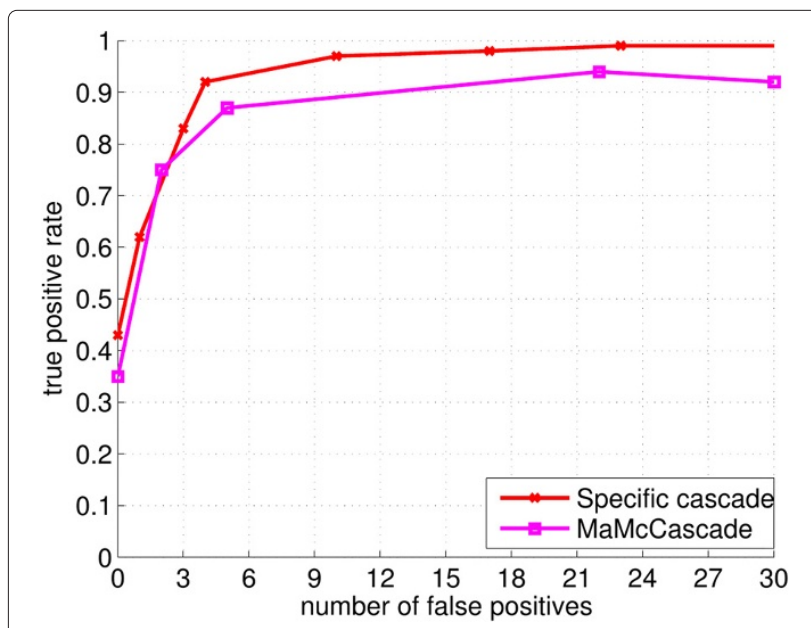

Figure 25 Comparison of the classifier MaMcCascade and the specific cascade on faces turned $45^{\circ}$.
Table 3 Mean detection time on faces turned $45^{\circ}$

\begin{tabular}{lccc}
\hline Classifier & $\begin{array}{c}\text { Mean time } \\
(\mathbf{m s})\end{array}$ & $\begin{array}{c}\text { Minimum time } \\
(\mathbf{m s})\end{array}$ & $\begin{array}{c}\text { Maximum time } \\
(\mathbf{m s})\end{array}$ \\
\hline Cascade & $234 \pm 46$ & 196 & 593 \\
MaMcCascade & $296 \pm 96$ & 201 & 663
\end{tabular}

Times for the initial upright face detector (noted Cascade) and for the MaMcCascade system are compared.

- $\mathcal{C}^{22,5}$ has, on average, $18 \%$ unavailable weak classifiers per level.

- $\mathcal{C}^{45}$ has, on average, $27 \%$ unavailable weak classifiers per level.

- $\mathcal{C}^{67,5}$ has, on average, $44 \%$ unavailable weak classifiers per level.

Unlike using the naive approach to handle these unavailable weak classifiers, it could be interesting to modify the cascade structure into a McCascade. In this section, the structure of the three detectors is changed into a McCascade. The strategy $P_{\mathrm{knn}}$ is used with $k=3$ neighbors, and thresholds $\beta_{j}$ are fixed using the cost function TP_cost. In Figures 23 and 24, these detectors are noted 'MaMcCascade.' On faces turned $22.5^{\circ}$ and $45^{\circ}$, the improvement compared to the naive approach is slight (increase of the detection rate from $2 \%$ to $5 \%$ ). The impact of using a McCascade is greater on faces turned $67.5^{\circ}$. Indeed, contrary to the naive approach, the McCascade allows for the detection rate to be improved with only a few false-positives. However, performances remain limited. For example, $55 \%$ of faces are detected with 12 falsepositives, while this rate is $90 \%$ when faces are turned $22.5^{\circ}$ and $45^{\circ}$.

Detecting faces turned $67.5^{\circ}$ with the existing solutions such as $[1,12,13]$ will exhibit better results. Indeed, a specific classifier will be train to detect faces turned $67.5^{\circ}$. When faces are turned $45^{\circ}$, it is interesting to compare the system MaMcCascade with a specific classifier. Thus, a specific classifier was trained using the same training parameters as the cascade $\mathcal{C}$, except that the positive images were extracted from the FERET database. A total of 132 images of faces turned $45^{\circ}$ were extracted to train the specific classifier (these images are not used during the testing stage). Results can be found in Figure 25 where we

Table 4 Mean detection time on faces occluded by a scarf

\begin{tabular}{lccc}
\hline Classifier & $\begin{array}{c}\text { Mean time } \\
\text { (ms) }\end{array}$ & $\begin{array}{c}\text { Minimum time } \\
\text { (ms) }\end{array}$ & $\begin{array}{c}\text { Maximum time } \\
\text { (ms) }\end{array}$ \\
\hline Cascade & $375 \pm 43$ & 272 & 610 \\
McCascade + evidence & $468 \pm 63$ & 335 & 758 \\
\hline
\end{tabular}

Times for the initial upright face detector (noted Cascade) and for the cascade associated with a McCascade (McCascade + evidence) are compared. 
see that the specific classifier gets a higher detection rate (up to $10 \%$ ).

\subsubsection{The multiview system}

In the previous sections, the pose of faces was known. Here, a multiview system is evaluated. This system can detect faces with different ROP angles. The three detectors $\mathcal{C}^{22,5}, \mathcal{C}^{45}$, and $\mathcal{C}^{67,5}$ are combined to get the multiview system following the principle of Section 5.2. Unavailable weak classifiers are handled with a McCascade. In Figures 23 and 24, this detector is noted 'MaMcCascade multiview'. It gets performances that are close to performances of specific detectors (noted MaMcCascade on each curve).

\subsection{Computation time}

In this section, we compare the execution time of the proposed method on the two applications. For the multiview application, we compare the initial upright face detector and the system MaMcCascade on faces turned $45^{\circ}$. The mean detection time per image, the minimum detection time, and the maximum detection time can be found in Table 3. For the occluded face detection application, we compare the initial upright face detector (noted Cascade) with the system of the initial cascade associated with a McCascade with the principle of cascading with evidence (noted McCascade + evidence) on faces occluded by a scarf. Table 4 contains detection times of the two systems. In both applications, classifiers were run five times and detection times were averaged. In both tables, we see that averaged detection time increases by about $25 \%$ when we use our solution.

\section{Conclusions}

We have presented a solution for handling missing weak classifiers in a boosted cascade. Our method relies on a probabilistic formulation of the cascade structure and on the computation of posterior probability on each level. To make a decision on each level, thresholds have been introduced and are fixed through an iterative procedure that minimizes a cost function. All aspects of the proposed solution have been tested. Moreover, the method has been successfully applied to two specific applications which involve occluded faces. During experiments on occluded faces and on turned faces, we also discuss limitations of the proposed solution which are due to performance differences between weak classifiers. On the other hand, the main advantage of the proposed method is that it only uses an existing face classifier; additional training is not needed to detect occluded faces or faces in another pose. Future work will focus on the method's limitation on occluded faces. During experiments on occluded faces, we notice that the proposed solution can fail on some occlusion types because learned weak classifiers do not cover the face with the same performance. To alleviate this problem, we plan to modify the initial training by adding constraints to the weak classifier locations.

\section{Consent}

Consent was obtained from the persons appearing in Figures 20 and 22 used for this publication.

\section{Competing interests}

The authors declare that they have no competing interests.

\section{Acknowledgements}

We want to thank OSEO for supporting our work which is part of the Biorafale project aimed at detecting and recognizing dangerous fans in football stadiums.

\section{Author details}

${ }^{1}$ Institut Pascal, Université Blaise Pascal 24, avenue des Landais, Aubière cedex 63177, France. ${ }^{2}$ Limos, Université Blaise Pascal 24, avenue des Landais, Aubière cedex 63177, France.

Received: 2 July 2012 Accepted: 27 June 2013

Published: 25 October 2013

\section{References}

1. CHuang, H Ai, Y Li, S Lao, High-performance rotation invariant multiview face detection. Trans. Patt. Anal. Mach. Intell. 29(4), 671-686 (2007)

2. Maytal Saar-Tsechansky, Foster Provost, Handling missing values when applying classification models. J Mach Learn Res. 8, 1623-1657 (2007)

3. F Smeraldi, M Defoin-Platel, M Saqi, in Data Integration in the Life Science, Handling missing features with boosting algorithms for protein-protein interaction prediction. ed by. P Lambrix, G Kemp. Proceedings of the 7th International Conference, DILS 2010, Gothenburg, Sweden, August 25-27, 2010. Lecture Notes in Computer Science, vol 6254 (Springer, Berlin, 2010), pp. 132-147

4. A Globerson, S Roweis, in Proceedings of the 23rd International Conference on Machine Learning (ICML '06), June 2006. Nightmare at test time: robust learning by feature deletion (ACM New York, 2006), pp. 353-360

5. O Dekel, O Shamir, L Xiao, Learning to classify with missing and corrupted features. Mach. Learn. 81, 149-178 (2008)

6. J Chen, S Shan, S Yang, X Chen, W Gao, Modification of the adaboost-based detector for partially occluded faces. 18th Int. Conf. Pattern Recognit. 2, 516-519 (2006)

7. YY Lin, TL Liu, CS Fuh, Fast object detection with occlusions. Eur. Conf. Comput. Vis. 3021, 402-413 (2004)

8. RE Schapire, The strength of weak learnability. Mach. Learn. 5(2), 197-227 (1990)

9. PViola, M Jones, Rapid object detection using a boosted cascade of simple features. Conf. Comput. Vis. Pattern Recognit. 1, 511-518 (2001)

10. J Friedman, T Hastie, R Tibshirani, Additive logistic regression : a statistical view of boosting. Ann. Statist. 28, 337-407 (2000)

11. L Lefakis, F Fleuret, Joint cascade optimization using a product of boosted classifiers. Adv. Neural Inf. Process. Syst. 23, 1315-1323 (2010)

12. YY Lin, TL Liu, Robust face detection with multi-class boosting. Conf. Comput. Vis. Pattern Recognit. 1, 680-687 (2005)

13. H Schneiderman, T Kanade, A statistical method for $3 \mathrm{~d}$ object detection applied to faces and cars. Conf. Comput. Vis. Pattern Recognit. 1, 746-751 (2000)

14. C Huang, H Ai, B Wu, S Lao, Boosting nested cascade detector for multi-view face detection. Int. Conf. Pattern Recognit. 2, 415-418 (2004)

15. O Tuzel, F Porikli, P Meer, in IEEE Conference on Computer Vision and Pattern Recognition, 17-22 June 2007. Human detection via classification on Riemannian manifolds (IEEE Piscataway, 2007), pp. 1-8

16. O Tuzel, F Porikli, P Meer, in Proceedings of the 19th European Conference on Computer Vision, May 2006. Region covariance : a fast descriptor for detection and classification (Springer-Verlag Berlin, 2006), pp. 589-600 
17. GB Huang, M Ramesh, T Berg, E Learned-Miller, Labeled faces in the wild: a database for studying face recognition in unconstrained environments. Technical Report 07-49, University of Massachusetts (2007)

18. M Muja, DG Lowe, in VISAPP International Conference on Computer Vision Theory and Applications (VISAPP'09), Lisbon, 5-8 February 2009. Fast approximate nearest neighbors with automatic algorithm configuration (INSTICC Press Setubal, 2009), pp. 331-340

19. HA Rowley, S Baluja, T Kanade, Neural network-based face detection. Trans. Patt. Anal. Mach. Intell. 20, 23-38 (1998)

20. J Yao, JM Odobez, Fast human detection from joint appearance and foreground feature subset covariances. Comput. Vis. Image Understanding. 115, 1414-1426 (2011)

21. AM Martinez, R Benavente, The AR face database. Technical Report 24, The Ohio State University, (1998)

22. M Jones, P Viola, Fast multi-view face detection. Technical Report 96, Mitsubishi Electric Research Laboratories, (2003)

23. R Lienhart, A Kuranov, V Pisarevsky, Empirical analysis of detection cascades of boosted classifiers for rapid object detection. Pattern Recognit. 2781, 297-304 (2002)

24. PJ Phillips, H Moon, SA Rizvi, PJ Rauss, The FERET evaluation methodology for face recognition algorithms. Trans. Patt. Anal. Mach. Intell. 22(10), 1090-1104 (2000)

doi:10.1186/1687-5281-2013-55

Cite this article as: Bouges et al:: Handling missing weak classifiers in boosted cascade: application to multiview and occluded face detection. EURASIP Journal on Image and Video Processing 2013 2013:55.

\section{Submit your manuscript to a SpringerOpen ${ }^{\mathcal{O}}$ journal and benefit from:}

- Convenient online submission

- Rigorous peer review

- Immediate publication on acceptance

- Open access: articles freely available online

- High visibility within the field

- Retaining the copyright to your article

Submit your next manuscript at $\boldsymbol{\wedge}$ springeropen.com 